\begin{tabular}{|c|c|}
\hline Citation & $\begin{array}{l}\text { John Greenwood, Thanh Hai Phan, Yasuhiko Fujita, Zhi Li, Oleksandr } \\
\text { Ivasenko, Willem Vanderlinden, Hans Van Gorp, Wout Frederickx, Gang Lu, } \\
\text { Kazukuni Tahara, Yoshito Tobe, Hiroshi Uji-i, Stijn F. L. Mertens, and Steven } \\
\text { De Feyter } \\
\text { Covalent Modification of Graphene and Graphite Using Diazonium } \\
\text { Chemistry: Tunable Grafting and Nanomanipulation } \\
\text { ACS Nano, } 2015,9,5,5520 \text { - } 5535 .\end{array}$ \\
\hline Archived version & $\begin{array}{l}\text { Author manuscript: the content is identical to the content of the published } \\
\text { paper, but without the final typesetting by the publisher }\end{array}$ \\
\hline Published version & $\begin{array}{l}\text { insert link to the published version of your paper } \\
\text { http://dx.doi.org/10.1021/acsnano.5b01580 }\end{array}$ \\
\hline Journal homepage & $\begin{array}{l}\text { insert link to the journal homepage of your paper } \\
\text { http://pubs.acs.org/journal/ancac3 }\end{array}$ \\
\hline Author contact & $\begin{array}{l}\text { your email steven.defeyter@kuleuven.be } \\
\text { your phone number }+32(0) 16327921\end{array}$ \\
\hline IR & url in Lirias https://lirias.kuleuven.be/handle/123456789/525598 \\
\hline
\end{tabular}




\section{Covalent Modification of Graphene and Graphite Using Diazonium Chemistry: Tunable Grafting and Nano-Manipulation}

John Greenwood, ${ }^{1 \ddagger}$ Thanh Hai Phan, ${ }^{1 \ddagger}$ Yasuhiko Fujita, ${ }^{1}$ Zhi Li, ${ }^{1}$ Oleksandr Ivasenko, ${ }^{1 *}$ Willem Vanderlinden, ${ }^{1}$ Hans Van Gorp, ${ }^{1}$ Wout Frederickx, ${ }^{1}$ Gang Lu, ${ }^{1}$ Kazukuni Tahara, ${ }^{3}$ Yoshito Tobe,${ }^{3}$ Hiroshi Uji-i, ${ }^{1 *}$ Stijn F. L. Mertens, ${ }^{1,2}$ Steven De Feyter ${ }^{1 *}$

${ }^{1}$ Department of Chemistry, Division of Molecular Imaging and Photonics, KU Leuven-University of Leuven, Celestijnenlaan 200F, B-3001 Leuven, Belgium.

${ }^{2}$ Vienna University of Technology, Institute of Applied Physics, Wiedner Hauptstraße 8-10/E134, A-1040 Vienna, Austria.

${ }^{3}$ Division of Frontier Materials Science, Graduate School of Engineering Science, Osaka University, Toyonaka, Osaka 560-8531, Japan,

${ }^{\ddagger}$ These authors contributed equally to this work

* Corresponding authors:

Oleksandr.Ivasenko@chem.kuleuven.be

Hiroshi.Ujii@chem.kuleuven.be

Steven.DeFeyter@chem.kuleuven.be 


\begin{abstract}
We shine light on the covalent modification of graphite and graphene substrates using diazonium chemistry under ambient conditions. We report on the nature of the chemical modification of these graphitic substrates, the relation between molecular structure and film morphology, and the impact of the covalent modification on the properties of the substrates, as revealed by local microscopy and spectroscopy techniques and electrochemistry. By careful selection of the reagents and optimizing reaction conditions, a high density of covalently grafted molecules is obtained - a result that is demonstrated in an unprecedented way by scanning tunneling microscopy (STM) under ambient conditions. By nano-manipulation, i.e. nanoshaving using STM, surface structuring and functionalization at the nanoscale is achieved. This manipulation leads to the removal of the covalently anchored molecules, regenerating pristine $\mathrm{sp}^{2}$ hybridized graphene or graphite patches, as proven by space-resolved Raman microscopy and molecular self-assembly studies.
\end{abstract}

\title{
Keywords
}

Graphene, STM, AFM, Raman spectroscopy, Diazonium, Grafting, Self-assembly.

Graphene, a single atom thick sheet of $\mathrm{sp}^{2}$ hybridized carbon, is the focus of intense research efforts since its isolation by Novoselov et al. ${ }^{1}$ This is due to its unique electronic, optical, mechanical, and thermal characteristics. Despite these exceptional qualities, however, some inherent characteristics of graphene preclude its widespread use in technological applications. For example, the gapless band structure of pristine graphene leads to low on/off ratios in field effect transistors (FET), resulting in large static power dissipation, ${ }^{2}$ and, consequently, hindering the development of graphene based devices. Also, other carbon based sheet-like materials such as multilayered graphene and graphite attract a lot of interest. 
To take full advantage of the properties of these two-dimensional (2D) and layered carbon based materials, it is important to develop efficient chemical functionalization protocols. For instance, chemical defects in the $\mathrm{sp}^{2}$ hybridized surface lattice of graphene are known to promote bandgap opening, ${ }^{3-5}$ one of the requirements for the efficient operation of field-effect transistors. Developing protocols for band-gap opening of graphene through chemisorption of organic species should provide a high degree of control, through concentration variation and molecular design, in a way in which the current state of the art methods do not. The impact of functionalization is not limited to electronics. Chemical functionalization of graphene and graphite by covalent grafting is relevant for a broad range of applications; for example, sensors, ${ }^{6-7}$ and improved graphene solubility. ${ }^{8}$ New functionality is also anticipated when this covalent grafting can be achieved in a (nano) structured fashion. ${ }^{4,9-10}$

Graphene has a relatively low chemical reactivity; the electronic structure is such that the $\pi$ electrons are fully delocalized over the entire 2D network. Graphite, and in particular highly oriented pyrolytic graphite (HOPG), a high quality form of graphite, is even less reactive. ${ }^{4,11-14}$ Therefore, in order to perform chemical modifications, only very reactive reagents will suffice. Covalent modification of graphene and HOPG from radicals produced from diazonium reagents is the most common method of covalent modification. The mechanism involves the transfer of a delocalized electron from the carbon surface to the diazonium cation, and results in the release of a $\mathrm{N}_{2}$ molecule and the formation of a radical species. Subsequently, the radical species attacks a $\mathrm{sp}^{2}$ hybridized carbon lattice atom on the surface forming a covalent bond and converting it into $\mathrm{sp}^{3}$. The degree to which actual covalent attachment, as opposed to physisorption, takes place, however, is still disputed, ${ }^{15}$ as only very few methods directly probe the covalent bond. 
For many applications, including the significant bandgap opening of graphene, a high density of surface defects created through covalent bond formation is required. However, a major shortcoming of diazonium chemistry is the limited covalent grafting density in combination with multilayer formation or dendritic growth. ${ }^{4}{ }^{16}$ An approach to maximize the grafting density and to minimize multilayer formation could be the introduction of bulky or protecting groups. Indeed, control over the thickness of the grafted layer has been achieved by introducing protecting groups into the diazonium reagents, such as silyl ${ }^{17}$ and hydrozone ${ }^{18}$ groups, or by using radical scavenger species, ${ }^{19}$ as was demonstrated on glassy carbon. These methods prevent further radical attack to pre-grafted species and limits growth of the grafted layer to a monolayer. Hence, limiting aryl growth to a single molecular layer is possible by the introduction of sterically hindering groups into the precursor diazonium species. A variety of bulky diazonium species have been grafted on a range of non-graphitic surfaces. ${ }^{13-14}$

For applications where grafted molecules are to be removed locally, a possible approach to form (nano) patterned surfaces, the question arises if such can be done in a way that the intrinsic properties of graphene or graphite are restored, akin to the removal of thiol-based self-assembled monolayers on gold. ${ }^{20}$ Nanopatterning of graphene via nanoshaving of covalently grafted molecules and hydrogen has been demonstrated under ultrahigh vacuum (UHV) conditions. ${ }^{4,10}$ However, nanopatterning of grafted graphene has not yet been achieved under ambient conditions.

Consequently, there is a pressing need for high-density grafting of graphene and other graphitic substrates, without multilayer formation. Furthermore, reliable protocols must be developed for the controlled nanopatterning of covalently grafted graphene and other graphitic substrates. Another challenge is the quantitative evaluation of the grafting density. 
In this study, we compare the relation between molecular structure and grafting morphology and density onto graphite and graphene of 4-nitrobenzenediazonium (4-NBD) ${ }^{3-5,21-24}$ tetrafluoroborate and bulky 3,5-bis-tert-butylbenzenediazonium (3,5-TBD), see Figure 1 for the respective chemical structures and reaction schemes. Both compounds are expected to form aryl radicals via electrochemical reduction and to graft onto these substrates, but their different structure should lead to different film morphologies. We demonstrate using atomic force microscopy (AFM) and scanning tunneling microscopy (STM) that the bulky tert-butyl groups on 3,5-TBD prevent multilayer polyaryl film growth, limiting grafting to monolayer coverage, and leading to a high surface density of the grafted species. Furthermore, STM allows for the first time to visualize and address individual covalent grafting sites in ambient conditions on previously defect-free $\mathrm{sp}^{2}$ carbon surfaces. Using scanning probe microscopy based nano-manipulation protocols under ambient conditions we can remove the grafted layer. Importantly, we prove that this procedure regenerates the original $\mathrm{sp}^{2}$ pristine graphite surface, as proven by confocal Raman spectroscopy and molecular self-assembly. 
a)

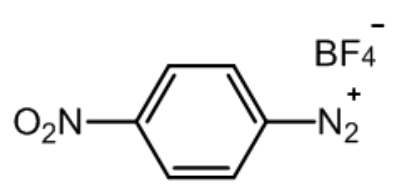

b)

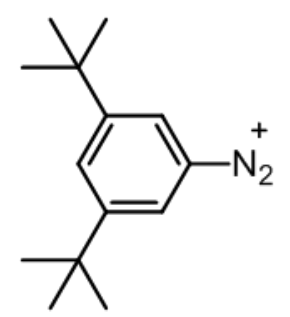

c)

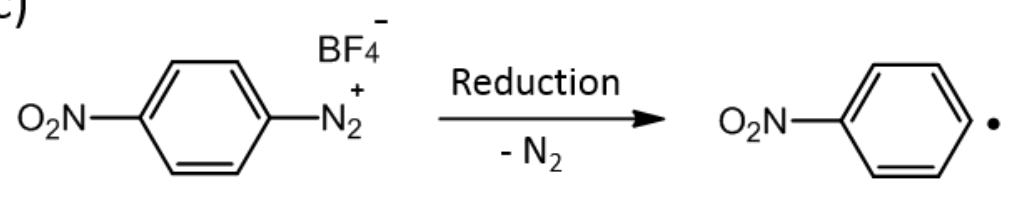

d)
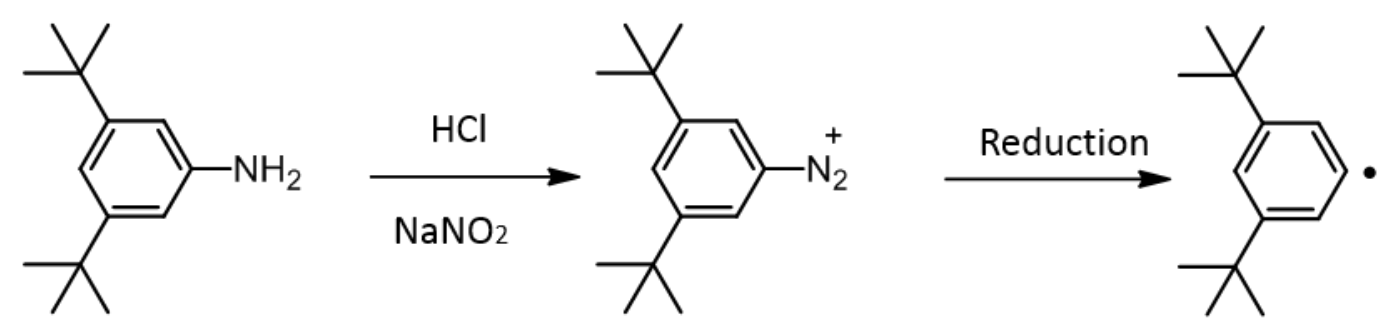

Figure 1. Chemical structures of (a) 4-nitrobenzenediazonium (4-NBD) tetrafluoroborate and (b) 3,5-bis-tert-butylbenzenediazonium (3,5-TBD); reaction scheme (c) for the reduction of 4-NBD to a nitrophenyl radical, and (d) for the conversion of the amino group of 3,5-bis-tert-butylaniline to a diazonium group, and subsequent reduction to an aryl radical.

\section{Results and Discussion}

We have utilized a combination of complementary surface analytical techniques to gauge the quality and extent of covalent attachment (chemisorption) of aryl species on graphene and HOPG. Cyclic voltammetry provides a qualitative assessment of the grafting process. AFM gives topographical information on the surface deposits. STM visualizes the sites of covalent attachment 
and gives a reliable estimation of the surface coverage. Raman spectroscopy and microscopy provide information on the hybridization $\left(\mathrm{sp}^{2} v s \mathrm{sp}^{3}\right)$ of surface lattice sites.

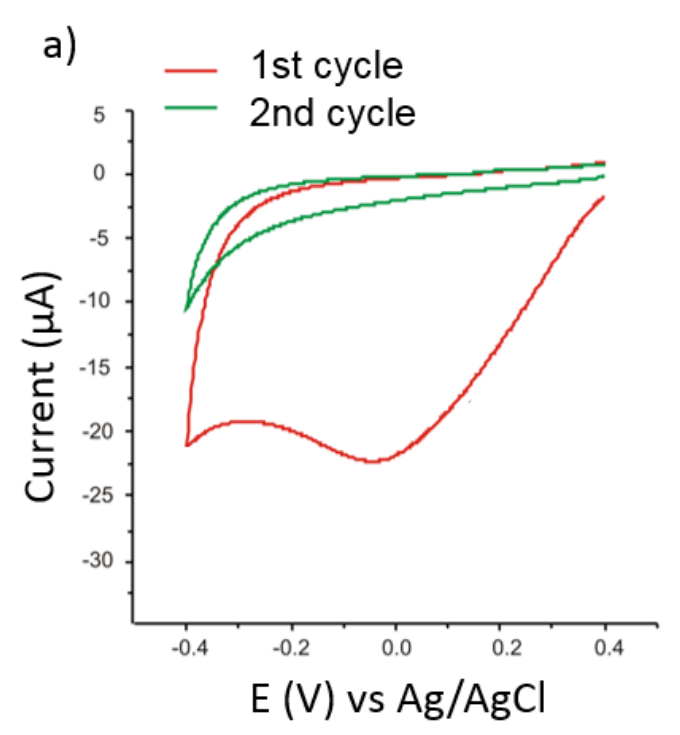

c)

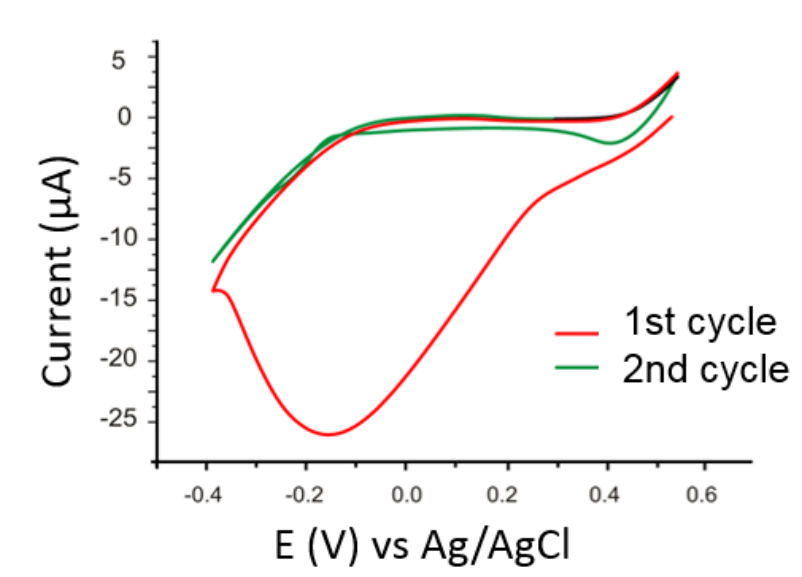

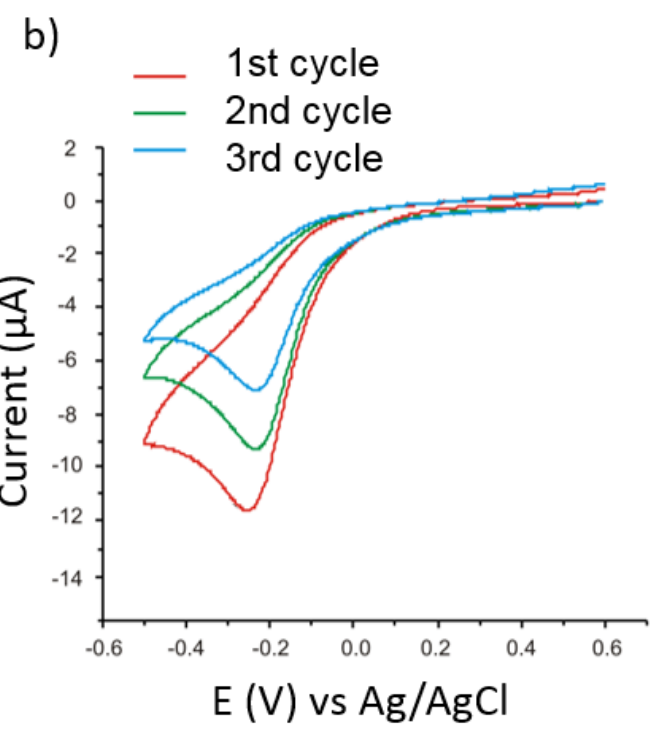

d)

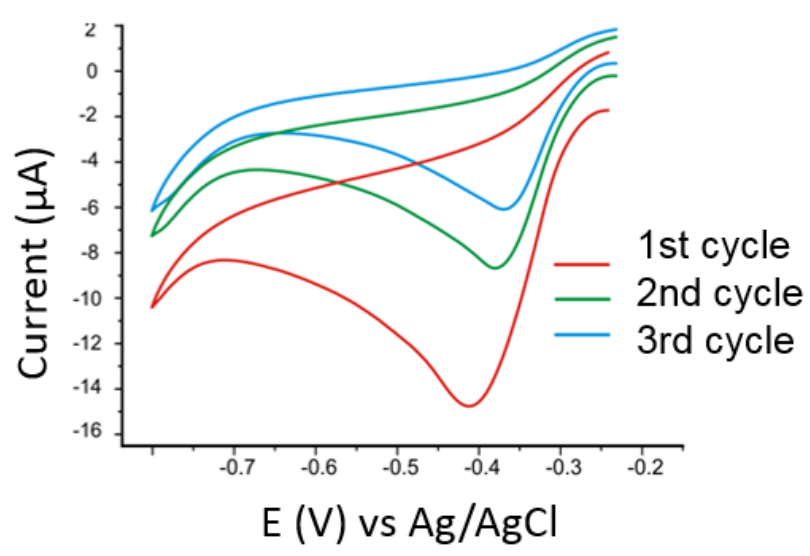

Figure 2. Cyclic voltammetry of (a) HOPG in $1 \mathrm{mM}$ 4-NBD, (b) HOPG in $1 \mathrm{mM}$ 3,5-TBD, (c) CVD graphene on $\mathrm{Cu}$ in $1 \mathrm{mM}$ 4-NBD, and (d) CVD graphene on $\mathrm{Cu}$ in $1 \mathrm{mM}$ 3,5-TBD. Supporting electrolyte $0.1 \mathrm{M} \mathrm{HClO}_{4}(\mathrm{a}, \mathrm{c})$ or $0.5 \mathrm{M} \mathrm{HCl}(\mathrm{b}, \mathrm{d})$. Scan rate $50 \mathrm{mV} \mathrm{s}^{-1}$. Potential limits: (a) 0.4 to $-0.4 \mathrm{~V}$, (b) 0.6 to $-0.5 \mathrm{~V}$, (c) 0.6 to $-0.4 \mathrm{~V}$, and (d) 0.6 to $-0.8 \mathrm{~V}$. 
Electrochemical surface modification of HOPG and graphene was carried out during cyclic voltammetry in aqueous solutions of the diazonium compounds. Detailed protocols are given in the Methods section. Figure 2a shows representative cyclic voltammograms (CVs) of HOPG exposed to aqueous $1 \mathrm{mM} 4-\mathrm{NBD}+0.1 \mathrm{M} \mathrm{HClO}_{4}$. The first cycle shows a broad irreversible peak around $E=-30 \mathrm{mV} v s \mathrm{Ag} / \mathrm{AgCl}$, which is assigned to the reduction of the 4-NBD cations and the formation of the corresponding aryl radicals. ${ }^{23,25}$ The second cycle is featureless, which suggests blocking of the surface by the products formed during the first cycle, on carbon ${ }^{23,25}$ and noncarbon ${ }^{13,26-27}$ surfaces alike. Analogous behavior is observed for 4-NBD modification of graphene, Figure 2c. The disappearance of the peak feature after the first cycle is often taken as evidence that covalent grafting has resulted in the formation of a non-conducting surface film, although physisorption could equally account for this. ${ }^{15}$

The electrochemical reduction of the sterically hindered 3,5-TBD on HOPG is shown in Figure 2b. Compared to 4-NBD (Figure 2a), the irreversible reduction peak has a well-defined shape and occurs at somewhat more negative potentials. Significantly, the current observed in subsequent cycles decreases more slowly than with 4-NBD, which may indicate a more gradual modification of the electrode surface, or that the surface modification does not block further electron transfer. Again, analogous behavior is observed for 3,5-TBD modification of graphene, Figure 2d.

Raman spectroscopy directly discloses information related to surface defects formed on carbon surfaces. The D-peak in the Raman spectra of graphene and HOPG is activated by lattice defects, including the formation of $\mathrm{sp}^{3}$ hybridization by covalent chemistry. ${ }^{28}$ The integrated intensity ratio of the D- and G-peaks $\left(\mathrm{I}_{\mathrm{D}} / \mathrm{I}_{\mathrm{G}}\right)$ is a measure of the concentration of covalent defect sites, and is a standard method to characterize the degree of covalent functionalization. ${ }^{3,24}$ Therefore, this 
technique has been used to determine the extent of $\mathrm{sp}^{3}$ hybridized defects formed after grafting with the diazonium reagents.

The Raman spectrum in Figure 3a is for 4-NBD grafted on HOPG from a $1 \mathrm{mM}$ solution. Note that all Raman spectra in this section were averaged over 5 sample spots. Raman peaks are observed at 1576, 2458, 2629, and $2679 \mathrm{~cm}^{-1}$. The intense peaks at 1576 and $2679 \mathrm{~cm}^{-1}$ are assigned to the G-band and 2D-band, respectively. The additional band at $1336 \mathrm{~cm}^{-1}\left(\mathrm{I}_{\mathrm{D}} / \mathrm{I}_{\mathrm{G}}=0.006\right)$ corresponds to the D-band, which can only be activated where a structural disorder is present in the $\mathrm{sp}^{2}$ carbon lattice, ${ }^{29}$ shown in the inset in Figure 3a. The D-band intensity after grafting from a $1 \mathrm{mM}$ solution of 4-NBD is extremely low, indicating a very low number of $\mathrm{sp}^{3}$ defects in the carbon surface due to grafting. If grafting is performed from 4-NBD solutions with concentrations above $1 \mathrm{mM}$, there is no further increase in the $\mathrm{I}_{\mathrm{D}} / \mathrm{I}_{\mathrm{G}}$ value. No D-band is observed after grafting from 0.01 and $0.1 \mathrm{mM}$ 4-NBD solutions, which likely stems from too low a number of generated $\mathrm{sp}^{3}$ defects to produce a detectable D-band.

Figure $3 \mathrm{~b}$ shows the Raman spectra after grafting of 3,5-TBD on HOPG from solutions containing $0.1,0.3,0.6,1,2$, and $5 \mathrm{mM}$, as well as for bare HOPG. Bands are observed at: 1330, 1578, and $2674 \mathrm{~cm}^{-1}$. The intensity ratios of the D- to G-bands are as follows: $0.1 \mathrm{mM}\left(\mathrm{I}_{\mathrm{D}} / \mathrm{I}_{\mathrm{G}}=0.0004\right), 0.3$ $\mathrm{mM}\left(\mathrm{I}_{\mathrm{D}} / \mathrm{I}_{\mathrm{G}}=0.047\right), 0.6 \mathrm{mM}\left(\mathrm{I}_{\mathrm{D}} / \mathrm{I}_{\mathrm{G}}=0.076\right), 1 \mathrm{mM}\left(\mathrm{I}_{\mathrm{D}} / \mathrm{I}_{\mathrm{G}}=0.086\right), 2 \mathrm{mM}\left(\mathrm{I}_{\mathrm{D}} / \mathrm{I}_{\mathrm{G}}=0.093\right)$, and 5 $\mathrm{mM}\left(\mathrm{I}_{\mathrm{D}} / \mathrm{I}_{\mathrm{G}}=0.1\right)$. Figure $3 \mathrm{c}$ shows that the relative D-band intensity at $1330 \mathrm{~cm}^{-1}$ increases for higher concentrations until it reaches a plateau at approximately $5 \mathrm{mM}$; the D-band intensity was found to be approximately the same as for a $10 \mathrm{mM}$ sample $\left(\mathrm{I}_{\mathrm{D}} / \mathrm{I}_{\mathrm{G}}=0.1\right.$, spectrum shown in supplementary information, Figure S4d), indicating that the D-band intensity maximum value occurs at $\sim 5 \mathrm{mM}$. In the case of 3,5-TBD, the D-band intensity obtained with a $1 \mathrm{mM}$ solution is 
relatively much larger than that observed for 4-NBD at the same concentration, as side reactions leading to polyaryl structures are not possible with 3,5-TBD.

The Raman spectrum for bare HOPG did not contain any D-band (see supplementary information, Figure S4a), indicating that the HOPG sample used did not contain any significant defects. Consequently, any D-band observed in Raman spectroscopy on grafted samples is the result of $\mathrm{sp}^{2}$-to-sp $\mathrm{s}^{3}$ rehybridization from aryl grafting to the HOPG surface. Importantly, we did not observe any D-band developing on bare HOPG as a result of scanning, excluding laser-induced damage at the power levels used.

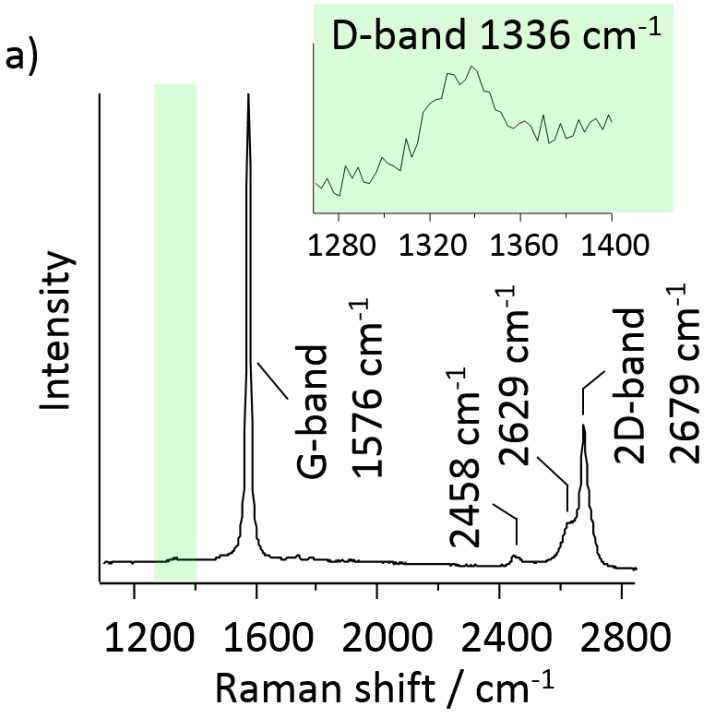



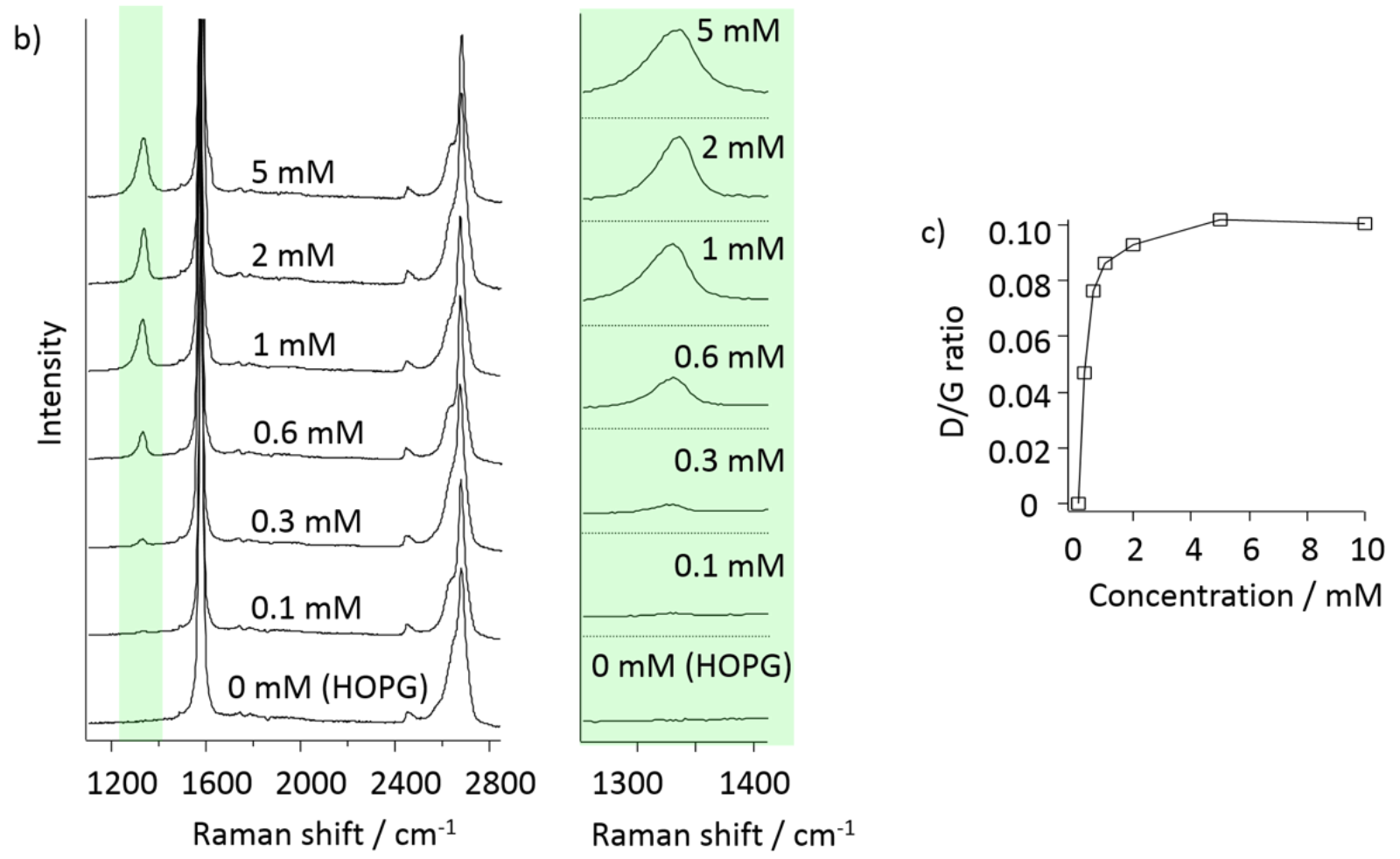

Figure 3. Raman spectra for (a) 4-NBD grafted on HOPG from a $1 \mathrm{mM}$ solution, and for (b) 3,5TBD grafted on HOPG from 0.1, 0.3, 0.6, 1, 2, and $5 \mathrm{mM}$ solutions, as well as for pristine HOPG. (c) D/G-band intensity versus 3,5-TBD concentration. The spectra were averaged over 5 sample spots. Integration time $100 \mathrm{~s}$.

Figure 4a shows the Raman spectrum for 4-NBD $1 \mathrm{mM}$ functionalized CVD graphene on $\mathrm{Cu}$. A very intense D-band is observed at $1332 \mathrm{~cm}^{-1}\left(\mathrm{I}_{\mathrm{D}} / \mathrm{I}_{\mathrm{G}}=1.14\right) .5 \mathrm{mM}$ 4-NBD modification resulted in a significantly lower D-band intensity compared to $1 \mathrm{mM}$, shown in supplementary information, (Figure S4e) and a white film was visible by eye on the graphene surface, even after washing with Milli-Q water. This is likely caused by extensive polyaryl formation or precipitation, decreasing the extent of grafting. The graphene samples used were of good quality, with a negligible degree 
of defect sites present on the pristine samples in the Raman spectra. Akin to HOPG, no physical damage was incurred from the Raman laser during scanning at the power levels used.

Figure $4 \mathrm{~b}$ shows the Raman spectrum after grafting of 3,5-TBD on graphene from solutions containing $0.1,0.3,0.6,1$, and $5 \mathrm{mM}$, as well as for pristine graphene. The main peak positions, as well as the $\mathrm{I}_{\mathrm{D}} / \mathrm{I}_{\mathrm{G}}$ and $\mathrm{I}_{2 \mathrm{D}} / \mathrm{I}_{\mathrm{G}}$ values are stated in Table 1. On increasing the concentration of 3,5$\mathrm{TBD}$, the $\mathrm{D}$ - and $\mathrm{D}$ '-band intensities increase; also, the 2D-band broadens and the intensity decreases. The behavior of increasing D-band growth for higher concentrations of 3,5-TBD on graphene closely correlates with that of grafting on HOPG. The D-band intensity reaches a maximum value $\left(\mathrm{I}_{\mathrm{D}} / \mathrm{I}_{\mathrm{G}}=3.1\right)$ for grafting with $3,5-\mathrm{TBD}$ of $5 \mathrm{mM}$ concentration. The intensity decrease and broadening for the 2D-band is associated with defect formation on graphene. A red shift is observed relative to pristine graphene for the D-, D'-, G-, and 2D-bands, highlighted in Figure 4c, which increases for higher 3,5-TBD concentrations. The trend for the red shift of the D-, G-, and 2D-bands is shown in supplementary information, Figure S4f. In all cases, the gradient of the shift is initially large for 3,5-TBD concentrations of between 0.1 and $1 \mathrm{mM}$, then levels off at $5 \mathrm{mM}$.

As an important intermediate conclusion, the Raman spectroscopy data unambiguously indicates covalent attachment of the aryl radicals formed from the diazonium reagents to both HOPG and graphene. The larger intensity ratio of the D- and G-peaks for 3,5-TBD modified HOPG and graphene, compared to 4-NBD, indicates there is a greater extent of covalent functionalization for this species.

The red shift can be understood on the basis of the elongation of the carbon-carbon bonds after grafting, which are subsequently weakened and therefore lower their vibrational frequency. ${ }^{30}$ The red shifts indicate that the electronic properties of graphene are significantly affected by strain 
caused after grafting, breaking the sublattice symmetry of graphene. ${ }^{30-31} \mathrm{Ni}$ et al. have observed analogous results to our own of the red shifts of the G- and 2D-bands when they put graphene under controlled uniaxial strain by stretching the flexible substrate. ${ }^{30}$ In the same study, the authors calculated a band gap opening of the strained graphene at the $\mathrm{K}$ point of the Brillouin zone. The size of the band gap is shown to increase linearly with the increase of tensile strain, producing a band gap of $\sim 300 \mathrm{meV}$ for a strain of $1 \%$. We conclude, therefore, that grafting of graphene with 3,5-TBD results in a band gap opening, attributed to the breaking of sublattice symmetry of graphene under strain. ${ }^{30,32-34}$ Significantly, the extent of red shift of the G- and 2D-bands and, hence, of the band gap is tunable by concentration control of the diazonium precursor reagent.

For graphene FET applications, the minimum band gap to be comparable to silicon is $\sim 1 \mathrm{eV} .{ }^{35}$ So far, chemical functionalization has fallen short of this minimum requirement. Niyogi et al. used a combination of Raman spectroscopy and angle resolved photoemission spectroscopy (ARPES) to characterize nitrophenyl-grafted graphene samples. ${ }^{3}$ Both exfoliated and epitaxial graphene on SiC were subjected to covalent functionalization by 4-NBD and subsequent spectroscopic analysis, revealing the opening of a band gap of $\sim 0.4 \mathrm{eV} .^{3}$ The authors observed a considerable red shift of the G- $\left(22 \mathrm{~cm}^{-1}\right)$ and 2D-band $\left(71 \mathrm{~cm}^{-1}\right)$ following nitrophenyl-functionalization of epitaxial graphene. In contrast, the Raman spectrum in Figure 4a for 4-NBD modified graphene at saturation coverage shows a smaller red shift of the G- $\left(3 \mathrm{~cm}^{-1}\right)$, and 2D-band $\left(6 \mathrm{~cm}^{-1}\right)$ relative to the pristine graphene reference. The discrepancy is likely due to a different effect of the substrate. Indeed, the authors also demonstrated that nitrophenyl-functionalized exfoliated graphene showed a slight blue shift of the G- $\left(5 \mathrm{~cm}^{-1}\right)$ and 2D-bands $\left(1 \mathrm{~cm}^{-1}\right)$. Therefore, the much larger red shift for the same peaks for 3,5-TBD than for 4-NBD can be associated with a larger graphene band gap opening. 
Chemical doping is an effective method to tailor the electrical properties of graphene, and both ptype and n-type conductions are desired to construct complex logic circuits. Raman spectroscopy can be used to determine the extent of doping. The G-peak position blue shifts and its full width at half maximum (FWHM) decreases for both electron and hole doping. ${ }^{36}$ For both electron and hole doping, the peak intensity ratio $\mathrm{I}_{2 \mathrm{D}} / \mathrm{I}_{\mathrm{G}}$ ratio is known to decrease ${ }^{37}$ Experimentally, the behavior of the 2D-peak upon doping can be used to discriminate between electron and hole doping: its position remains almost unchanged for electron concentrations up to $\sim 1.5 \times 10^{13} \mathrm{~cm}^{-2}$, while it shifts down for larger concentrations. Conversely, an upshift is observed in case of hole accumulation. ${ }^{37}$ In principle, 3,5-TBD grafting to graphene could act as an electron dopant via $\sigma$ conjugation of the tert-butyl groups to the graphene substrate. However, the red shift in the Raman data shown here, caused by strain from defect formation, essentially neutralizes the ability to characterize doping by analyzing the G- and 2D- band shifts. The decrease in 2D-band intensity also cannot be used for doping characterization since it inherently decreases on defect formation. Consequently, there is no significant evidence of doping of graphene by grafting with 3,5-TBD. Grafting of 4-NBD to graphene ${ }^{38}$ and graphene nanoribbons (GNRs) ${ }^{39}$ is known to act as a pdopant; however, no evidence was observed in the Raman spectrum either, possibly also due to the strain caused by grafting, resulting in a red shift of the G- and 2D-bands. Nevertheless, we believe electron doping from grafting of 3,5-TBD to be viable, and to warrant further investigation. 

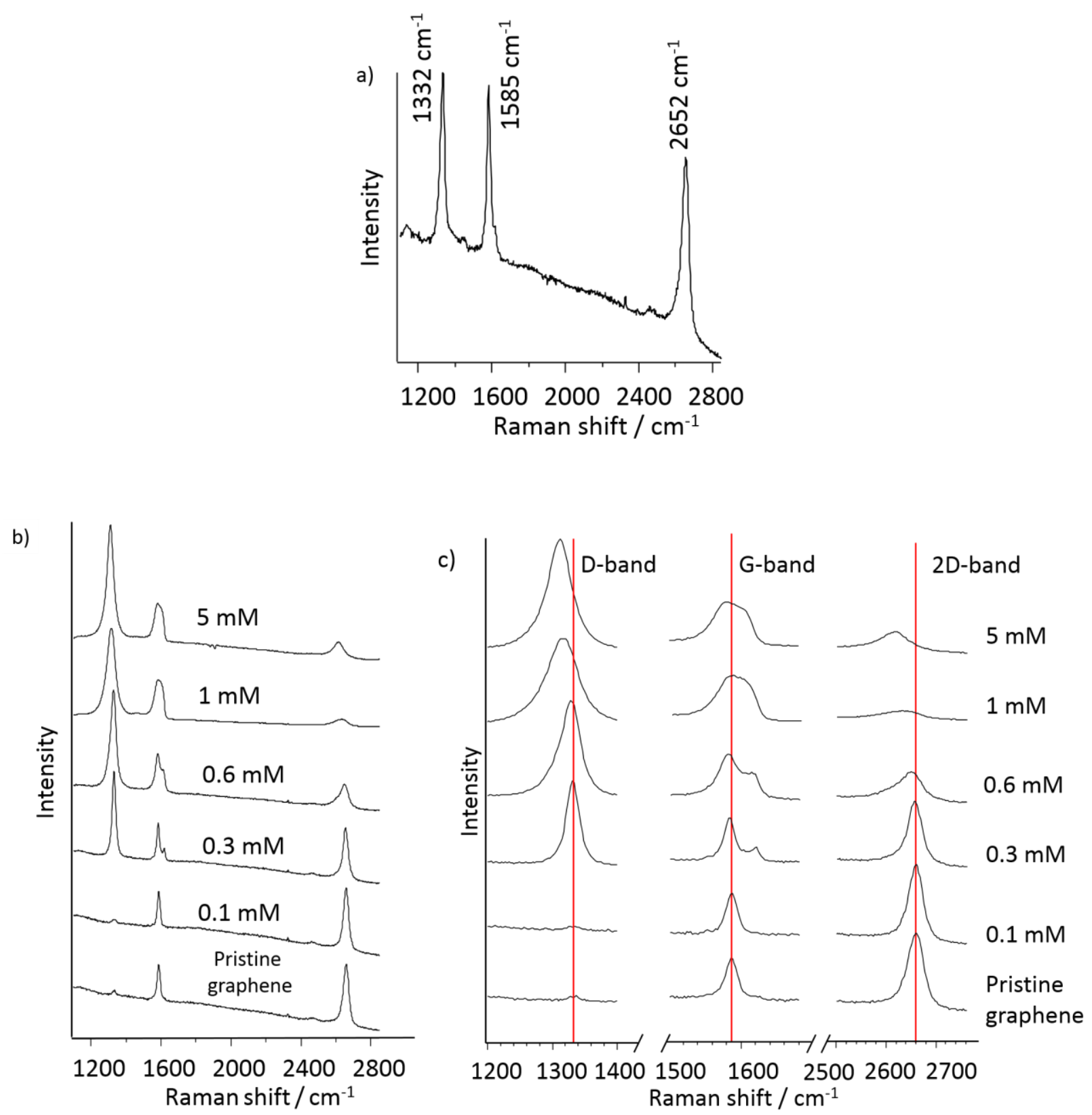

Figure 4. Raman spectra for CVD graphene on $\mathrm{Cu}$ after grafting of (a) 4-NBD from $1 \mathrm{mM}$ solution, and (b) 3,5-TBD from 0.1, 0.3, 0.6, 1, and $5 \mathrm{mM}$ solutions, as well as pristine CVD graphene on $\mathrm{Cu}$. Integration time $100 \mathrm{~s}$. The spectra are normalized with respect to $\mathrm{I}(\mathrm{G})$. (c) Raman spectra of the D-, G-, D'- (adjacent to G-band), and 2D-bands showing a red shift with respect to pristine graphene upon increasing 3,5-TBD concentration. 
Table 1. Raman spectra peak positions for a series of concentrations of 3,5-TBD grafted to graphene

\begin{tabular}{|c|c|c|c|c|c|c|}
\hline $\begin{array}{c}\text { Concentration } \\
\qquad(\mathbf{m M})\end{array}$ & $\mathbf{I}(\mathbf{D}) / \mathbf{I}(\mathbf{G})$ & $\mathbf{I}(\mathbf{2 D}) / \mathbf{I}(\mathbf{G})$ & $\begin{array}{l}\text { D-band } \\
\text { (cm-1) }\end{array}$ & $\begin{array}{l}\text { G-band } \\
(\mathbf{c m}-1)\end{array}$ & $\begin{array}{l}\text { D'-band } \\
(\mathrm{cm}-1)\end{array}$ & $\begin{array}{l}\text { 2D-band } \\
\text { (cm-1) }\end{array}$ \\
\hline $\begin{array}{l}0 \text { (Pristine } \\
\text { graphene) }\end{array}$ & 0.1 & 1.7 & 1333 & 1587 & No peak & 2658 \\
\hline 0.1 & 0.1 & 1.7 & 1333 & 1586 & No peak & 2658 \\
\hline 0.3 & 2.4 & 0.6 & 1331 & 1583 & 1618 & 2655 \\
\hline 0.6 & 2.7 & 0.6 & 1327 & 1581 & 1614 & 2646 \\
\hline 1 & 2.4 & 0.2 & 1315 & 1580 & 1605 & 2629 \\
\hline 5 & 3.1 & 0.4 & 1310 & 1579 & 1604 & 2614 \\
\hline
\end{tabular}

To gain further insight into the difference between 4-NBD and 3,5-TBD reactivity, we investigated the film morphology of grafted HOPG samples using AFM. The AFM images and line profiles of 4-NBD grafted HOPG from 0.01 and $1 \mathrm{mM}$ solutions are shown in Figures 5a and 5b, respectively. For the low concentration sample $(0.01 \mathrm{mM})$, bright patches are imaged along with large areas of 
bare surface. The height of the step between two bare surfaces is measured as $0.35 \mathrm{~nm}$, identical as for unmodified HOPG, suggesting the bare surface areas are pristine HOPG. Adsorbed material is also observed on the step edge in the image. The height profile of the features on the HOPG basal plane and step edge are measured as $2-3 \mathrm{~nm}$ (inset of figure 5a). To determine the height variation of the multilayer film, roughness analysis was performed using $\mathrm{WSxM},{ }^{40}$ which gave a root mean square (RMS) roughness of $0.53 \mathrm{~nm}$. The calculated height for a vertically oriented nitrophenyl group is $\sim 0.8 \mathrm{~nm} .{ }^{41}$ Therefore, the much larger height profile measured indicates that polyaryl growth already occurs at $0.01 \mathrm{mM}$ of 4-NBD. Consequently, polyaryl film growth must be kinetically very favourable and hinders high coverage of aryl radicals grafting directly to the HOPG surface. Figure 5b shows a much more densely packed structure formed from a $1 \mathrm{mM}$ solution of 4-NBD. The height profile gives a maximum value of $\sim 2.5 \mathrm{~nm}$ (see inset). This value is less than that observed for the lower concentration $(0.01 \mathrm{mM})$ sample, indicating that the line profile does not reach the surface; hence, no bare HOPG surface is exposed in this case. The RMS roughness value in this case is $0.34 \mathrm{~nm}$.

An AFM image for HOPG modified with 3,5-TBD $(0.01 \mathrm{mM})$ is shown in Figure 5c. Bright spots are observed on the surface. Height profile analysis (see inset) shows that the spots are quite uniform in height, at approximately $0.8-1 \mathrm{~nm}$, with an RMS roughness of $0.023 \mathrm{~nm}$, indicating a high degree of height uniformity. If a more concentrated solution is used ( $1 \mathrm{mM}$, Figure $5 \mathrm{~d})$, the image appears very uniform, confirmed by an RMS roughness of $0.085 \mathrm{~nm}$. In this case, no resolution of the individual adsorbed species was obtained due to tip convolution.

To accurately ascertain the organic film thickness at $1 \mathrm{mM}$ 4-NBD, scratching with the AFM tip was performed to expose the HOPG surface, Figure 5e. The depth of the scratched line was measured as $3.6 \pm 0.5 \mathrm{~nm}$. This value is comparable to the thickness measured by Ma et al. of 3.5 
$\pm 1.3 \mathrm{~nm}$ for $4-\mathrm{NBD}$ electrodeposited on HOPG. ${ }^{15}$ These authors concluded that multilayer coverage occurs, based on the calculated height of $\sim 0.8 \mathrm{~nm}^{41}$ for a vertically oriented nitrophenyl (NP) group. This result also explains the disappearance of the reduction peak after one cycle for 4-NBD (1 mM) grafting on HOPG and graphene, since the thick polyaryl layer formed prevents further electron transfer. During grafting of $0.01 \mathrm{mM} 4-\mathrm{NBD}$, the bare surface parts allow futher electron transfer. After scratching with the AFM tip, there is a build-up of deposit at the edge of the scratched area. We conclude, therefore, that upon scratching with the AFM tip, the detached species are pushed to the edge and on top of the polyaryl layer. The accumulated species likely consist of aryl oligomers.

To confirm whether grafting of 3,5-TBD on HOPG is limited to a single layer, AFM tip scratching was also performed on the $1 \mathrm{mM}$ sample, Figure 5f. The scratched line depth was measured as $0.67 \pm 0.31 \mathrm{~nm}$. This corresponds to the height of a single 3,5-TBD molecule, confirming that grafting in this case is limited to a single molecular layer. Histogram data for AFM scratching is shown in the supplementary information, Figure S5. The observation of single layer growth explains why electrochemical reduction of 3,5-TBD remains possible during consecutive scans. 

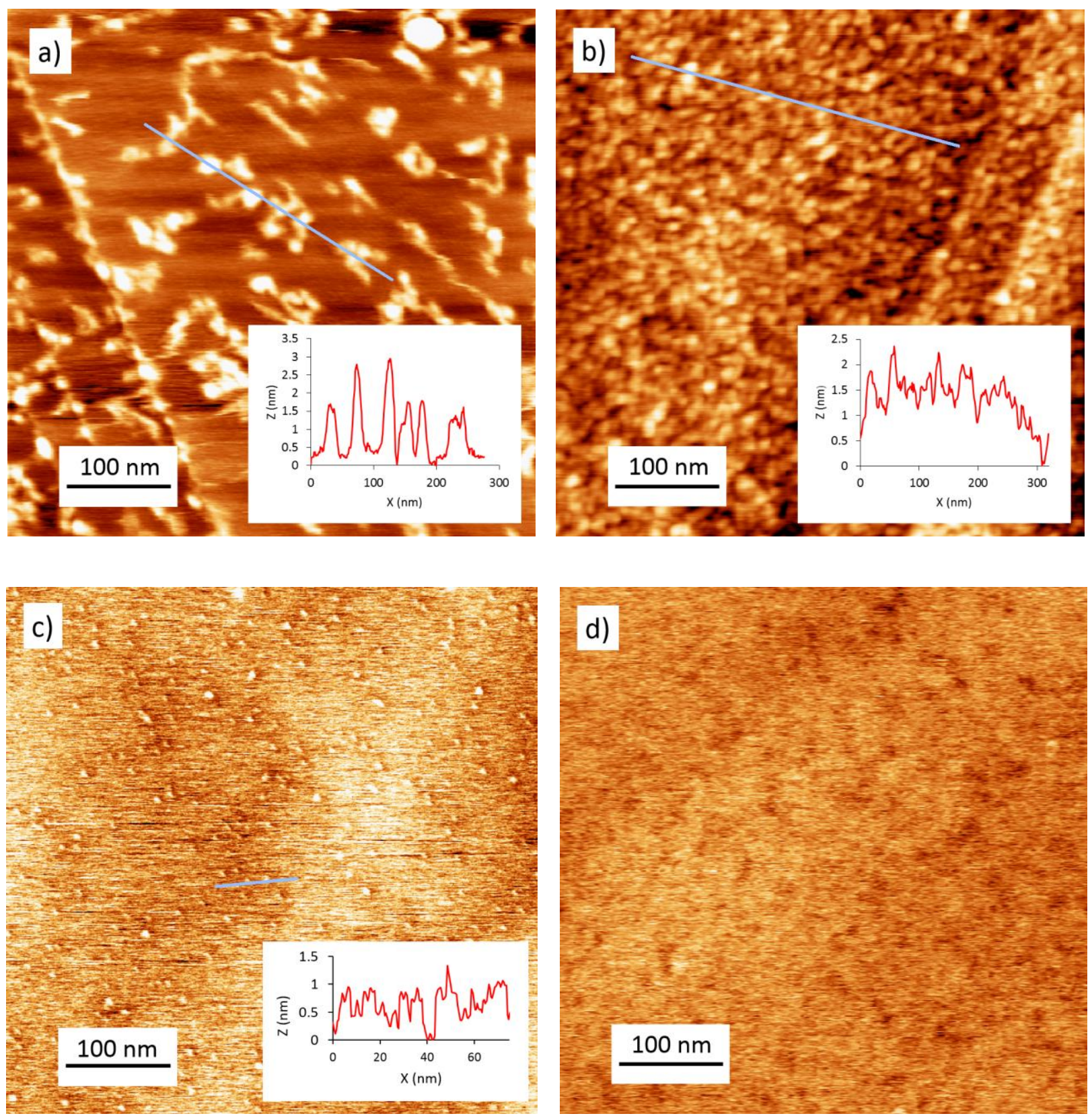

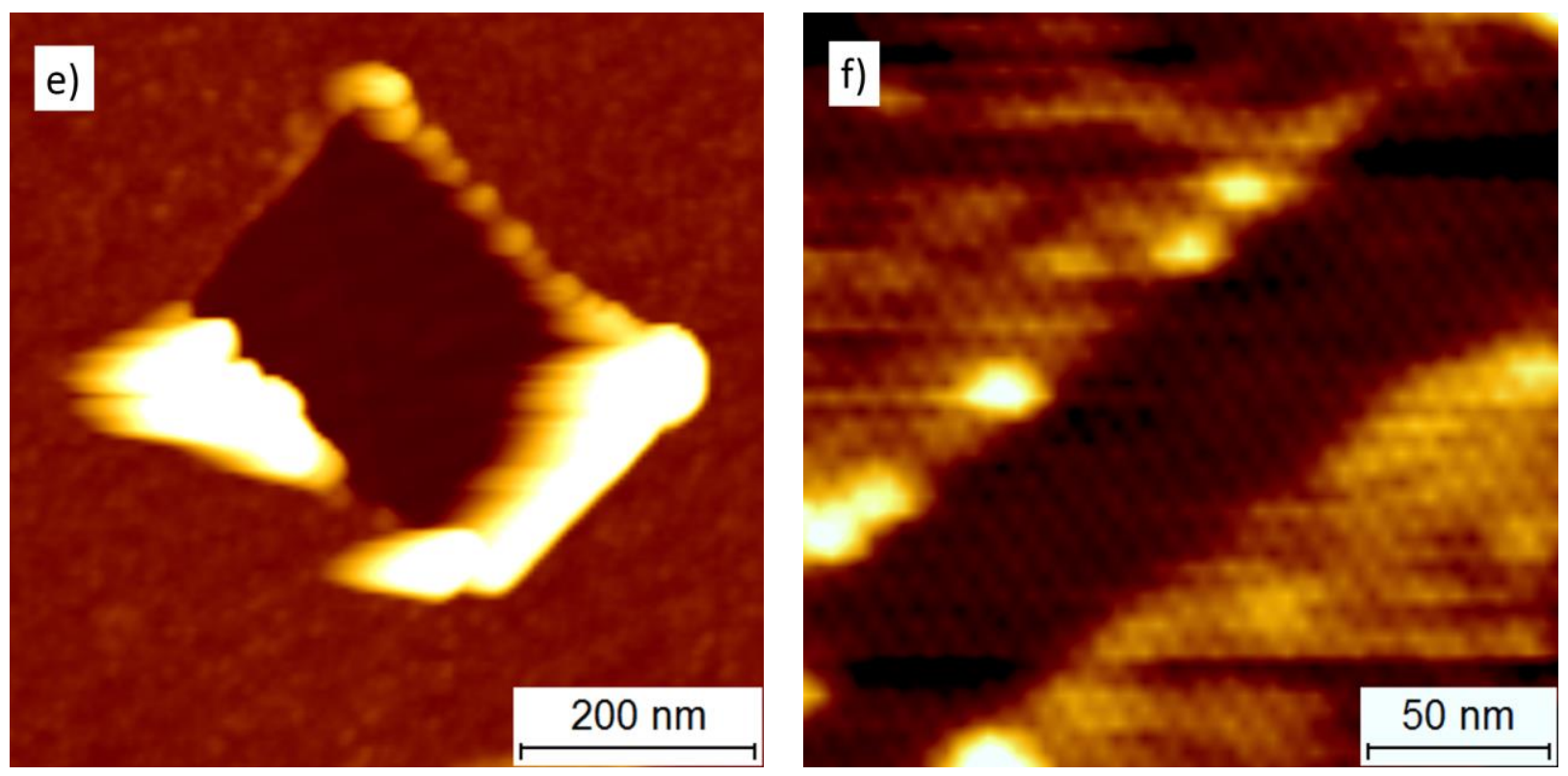

Figure 5. AFM images after grafting of 4-NBD on HOPG from (a) $0.01 \mathrm{mM}$ or (b) $1 \mathrm{mM}$ solutions, and after grafting of 3,5-TBD on HOPG from (c) $0.01 \mathrm{mM}$ or (d) $1 \mathrm{mM}$ solutions. AFM images after scratching the grafted layer with the AFM tip on (e) 4-NBD and (f) 3,5-TBD grafted HOPG, both from $1 \mathrm{mM}$ solutions.

While AFM is helpful in understanding film morphology, this technique is not ideal to quantitatively evaluate the density of covalent anchoring sites due to spatial resolution issues, in particular at high substrate coverage. STM, which is sensitive to the local density of states (LDOS) at the surface, may be more helpful here. The LDOS should be affected by the covalent modification, only at the grafting sites. Therefore, there is good hope that STM can reveal those sites.

As shown in Figure 6, image contrast very different from the AFM imaging is obtained. After grafting from $0.01 \mathrm{mM}$ 4-NBD (Figure 6a), bright features are observed at the step edges, with a height profile of approximately $1.5 \mathrm{~nm}$. Spots on the terraces are very few (Figure 6b), also with a height profile of $1.5 \mathrm{~nm}$. As shown in Figures $6 \mathrm{c}$ and $6 \mathrm{~d}$, the population of spots on the terraces is 
proportional to the concentration of the grafting solution. The variation in the appearance of the 4NBD modified sample in Figure 5b and 6d, visualized by AFM and STM, respectively, can be accounted for by the difference in the scanning mechanism of the two techniques. Since multilayer oligomer structures are formed following 4-NBD modification, AFM will visualize the topography of a broad top layer. STM, on the other hand, images mostly the covalent attachment points. The height profile of the bright STM features at all concentrations is comparable, at approximately 1.5 $\mathrm{nm}$, and was constant for the range of tunneling parameters used (setpoint current $0.05-0.15 \mathrm{nA}$ and bias voltage $0.4-0.9 \mathrm{~V}$ ). The measured spot width, however, is not constant for the range of concentrations used: at 0.01 and $0.1 \mathrm{mM}$, the average diameter of the spots is $1.6 \pm 0.1 \mathrm{~nm}$, whereas at $1 \mathrm{mM}$, the average diameter increases to $2.7 \pm 0.1 \mathrm{~nm}$. These values are too large to account for a single molecular species, and must consist of multiple molecules, referred to as clusters hereon. The lateral propagation at higher concentration is likely due to an increased reactivity of the lattice sites surrounding grafted molecules. The distribution of the clusters across the surface is not uniform, but displays a large variance. Statistical analysis of a $40,000 \mathrm{~nm}^{2}$ scan area of five different locations on each of three separate samples (grafting from $1 \mathrm{mM}$ solutions only) gave an average cluster density of 0.01 per $\mathrm{nm}^{2}$. 

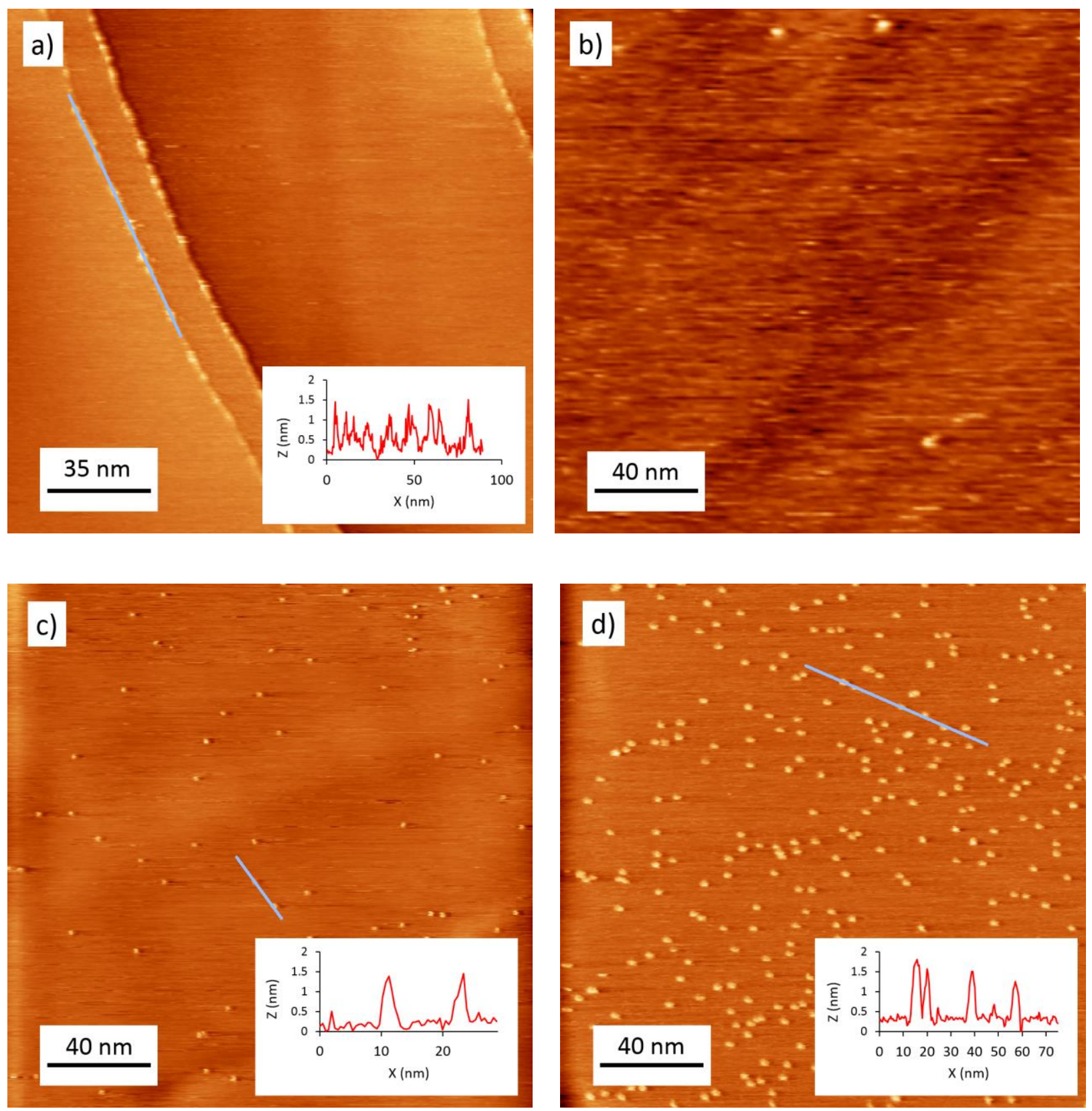

Figure 6. STM images after grafting of 4-NBD on HOPG from (a,b) $0.01 \mathrm{mM}$, (c) $0.1 \mathrm{mM}$, and

(d) $1 \mathrm{mM}$ solutions. Line profiles are shown as insets. Tunneling parameters $(\mathrm{a}, \mathrm{b}) \mathrm{I}_{\mathrm{set}}=0.08 \mathrm{nA}$, $\mathrm{V}_{\text {bias }}=-0.5 \mathrm{~V}$; (c) $\mathrm{I}_{\text {set }}=0.3 \mathrm{nA}, \mathrm{V}_{\text {bias }}=-0.4 \mathrm{~V}$; (d) $\mathrm{I}_{\text {set }}=0.08 \mathrm{nA}, \mathrm{V}_{\text {bias }}=-0.6 \mathrm{~V}$. 
We have also used STM to characterize grafted 3,5-TBD on HOPG. At 0.01 mM (Figure 7a), bright spots are observed on the step edges and on the terraces; however, the coverage on the terrace sites is extremely low. On increasing the 3,5-TBD concentration to $0.1 \mathrm{mM}$ (Figure 7b), the coverage of bright spots increases significantly. At $1 \mathrm{mM}$ (Figure 7c), a very high density of bright spots is observed. The density of the adsorbed species at $1 \mathrm{mM}$ is clearly higher than that observed for 4-NBD at the same concentration on HOPG, at $0.9 \pm 0.1$ clusters per $\mathrm{nm}^{2}$. This also correlates with the higher D-band intensity observed in the Raman spectrum for 3,5-TBD. After grafting from a $2 \mathrm{mM}$ solution (Figure 7d), the density of grafted structures is so high that tip convolution prevents the acquisition of the cluster density. STM images after grafting from even higher concentrations up to $10 \mathrm{mM}$ show similarly densely packed structures (Figure S3) in agreement with a levelling off in the intensity ratio of the D/G bands in the Raman spectra at these concentrations (see above). Similar to the behavior of 4-NBD, the density of spots increases with higher concentration following modification of HOPG with 3,5-TBD. The height profile of the spots for all concentrations is measured as $0.7-1 \mathrm{~nm}$. The value correlates well with the height profile of the spots measured with AFM; hence, the height profiles measured with STM confirm that the observed spots are a single molecule in height. In contrast to 4-NBD, the width of the spots for 3,5-TBD stays constant for the concentration range $0.01-1 \mathrm{mM}$, measured as $2.1 \pm 0.1$ nm. Analogous to 4-NBD, this value must consist of clusters of molecules, being too large to account for a single molecule. 

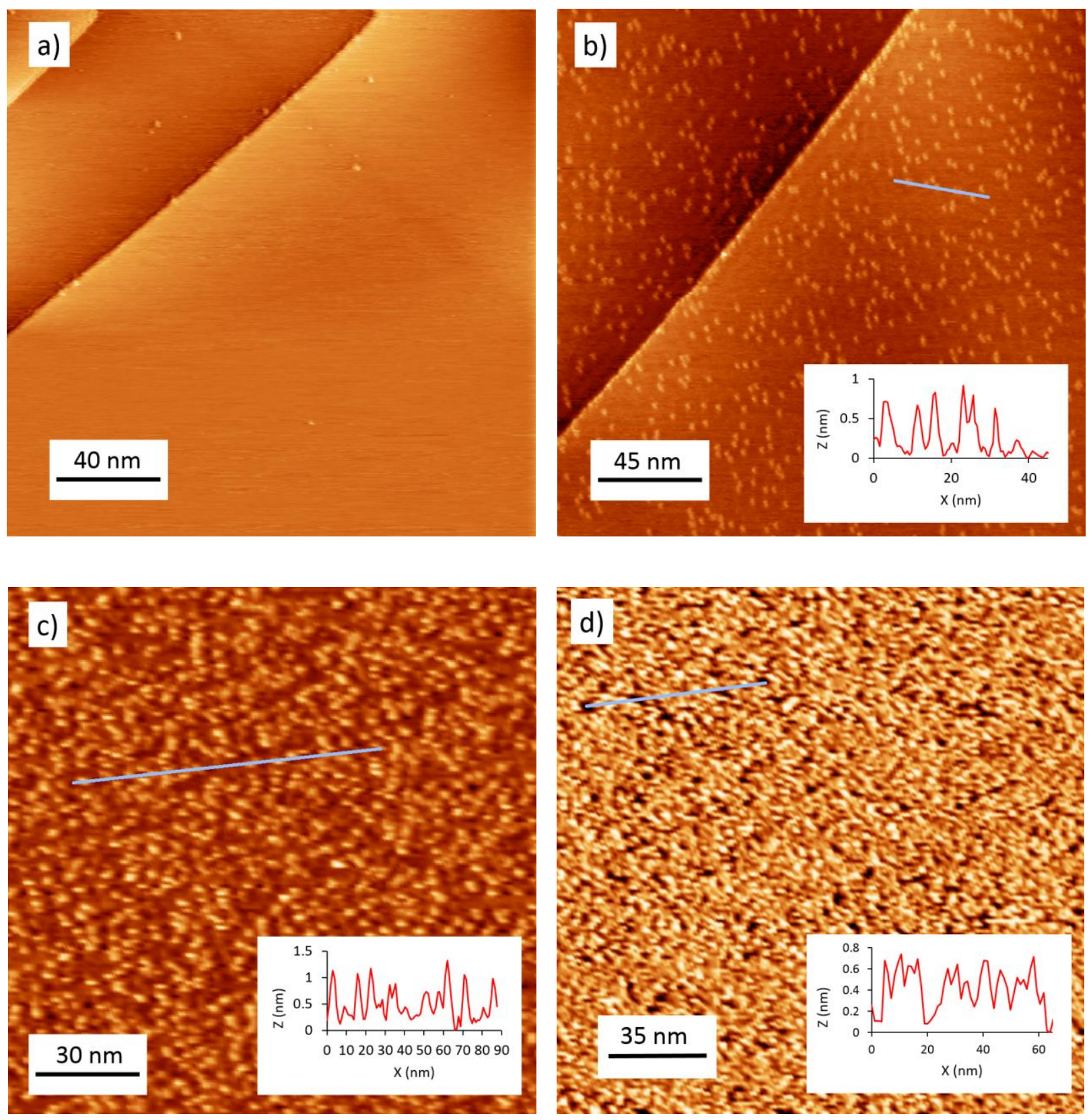

Figure 7. STM images after grafting of 3,5-TBD on HOPG from (a) $0.01 \mathrm{mM}$, (b) $0.1 \mathrm{mM}$, (c) 1 $\mathrm{mM}$, and (d) $2 \mathrm{mM}$ solutions. Line profiles are shown as insets. Tunneling parameters (a,b) $\mathrm{I}_{\mathrm{set}}=$ $0.08 \mathrm{nA}, \mathrm{V}_{\text {bias }}=-0.6 \mathrm{~V}$; (c) $\mathrm{I}_{\text {set }}=0.1 \mathrm{nA}, \mathrm{V}_{\text {bias }}=-0.4 \mathrm{~V}$; (d) $\mathrm{I}_{\text {set }}=0.16 \mathrm{nA}, \mathrm{V}_{\text {bias }}=-0.7 \mathrm{~V}$. 
STM images for 4-NBD and 3,5-TBD modified CVD graphene on $\mathrm{Cu}$ are shown in Figures 8a and b, respectively. Bright features are observed on both samples, whose coverage and distribution are analogous to those observed on HOPG. Again, the surface coverage after functionalization of graphene with 3,5-TBD is much higher than with 4-NBD, in agreement with the larger D/G band intensity in the Raman spectrum, described previously. Also the height profiles of the clusters on graphene, 1-1.5 nm following modification with 4-NBD (Figure 8a), and 0.8-1 nm for grafted 3,5-TBD (Figure 8b), are in agreement with values found on HOPG.
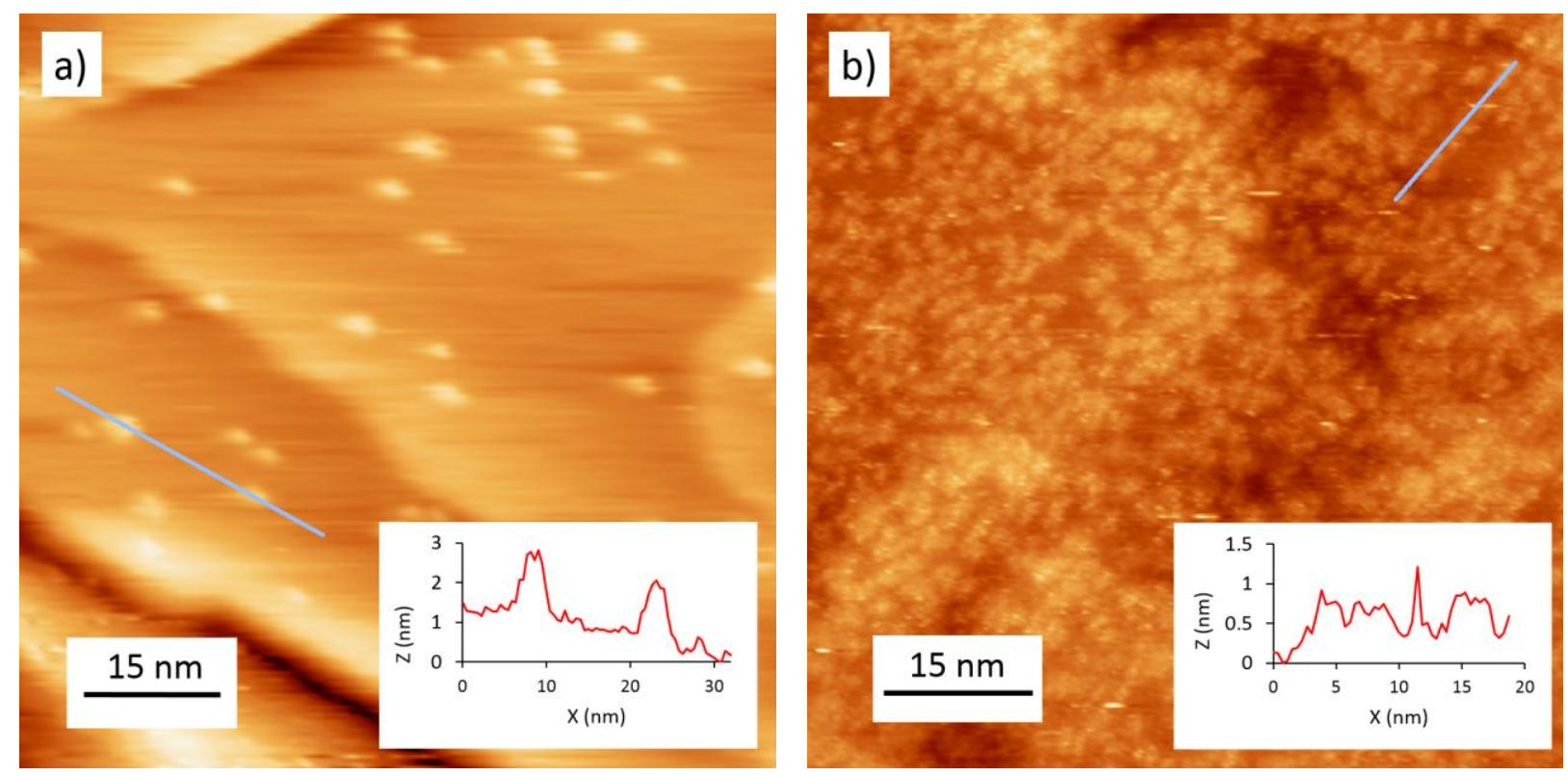

Figure 8. STM images of clusters after grafting from a $1 \mathrm{mM}$ solution of (a) 4-NBD and (b) 3,5TBD on CVD graphene on $\mathrm{Cu}$. Tunneling parameters $\mathrm{I}_{\text {set }}=0.08 \mathrm{nA}$ and $\mathrm{V}_{\text {bias }}=-0.7 \mathrm{~V}$.

All experimental evidence on HOPG and CVD graphene on $\mathrm{Cu}$ indicates that grafting of 4-NBD results in polyaryl growth. Following initial covalent attachment of aryl species to the surface, aryl radicals in solution can form covalent bonds at the ortho position with respect to the nitro group. Based on AFM data, polyaryl growth is found to already occur in the lowest concentration regime, 
at $0.01 \mathrm{mM}$. Therefore, radical attachment to pre-grafted aryl species must be kinetically very favorable. Consequently, every grafted (poly)aryl species functions as an ultra-microelectrode surrounded by a hemispherical diffusion layer, ${ }^{42}$ limiting the number of nitrophenyl species directly grafted to the surface. In contrast, grafting of 3,5-TBD leads to monolayer growth, as the tert-butyl moieties prevent further radical attachment to pre-grafted species and therefore preclude multilayer growth. This enables a large proportion of the radicals in solution to graft directly to the surface, resulting in high density grafting. Figure 9 illustrates this concept.
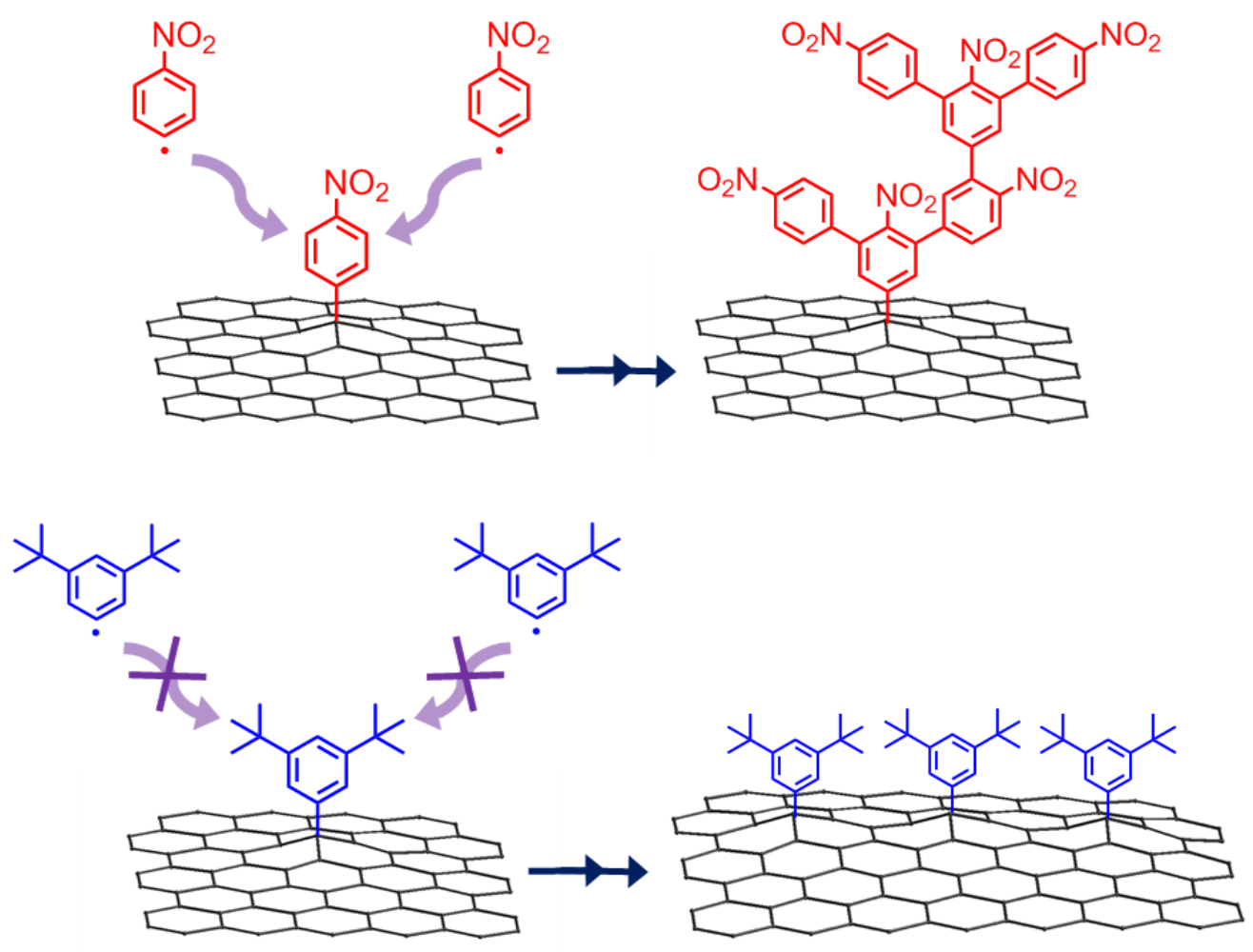

Figure 9. Grafting behavior of 4-NBD and 3,5-TBD, showing dendritic growth or monolayer formation.

In addition to the size of the bright spots, the so-called clusters, that are larger than the dimensions expected for individual grafting sites, STM images reveal another interesting aspect. The image in 
Figure 10a shows the 4-NBD clusters imaged along with the graphite lattice. It is apparent that there is a distortion to the graphite lattice in the vicinity of the clusters. The diameter of the distortions is not constant, and ranges from 8 to $2 \mathrm{~nm}$. The height profile of the clusters is $0.4-$ $0.8 \mathrm{~nm}$. This value is significantly lower than that measured for the clusters in the previous images at different scanning parameters. The reason for the height difference could be a change in the measured LDOS of the polyaryl clusters at the scanning parameters used for imaging the graphite lattice. The cluster diameter is $\sim 2.5 \mathrm{~nm}$, similar to those in the previous images. The lateral size of the bright spots is in line with theoretical studies that suggest that radical attachment to the carbon lattice occurs in pairs. ${ }^{29,30}$ Therefore, the cluster formation observed is likely due to further aryl radicals attacking the carbon lattice at the periphery of the nucleation sites. In addition, upon the attachment of an aryl pair, the rehybridization of the carbon lattice atoms to $\mathrm{sp}^{3}$ will distort the surrounding sites, causing buckling of the graphite lattice, which is picked up in the STM imaging of the graphite lattice. These sites are known to be more reactive, ${ }^{5}$ but further lateral propagation of the clusters is probably offset due to steric hindrance factors.

Direct high-resolution UHV-STM imaging of grafting has been attempted by Stevenson et al. ${ }^{43}$ but on a graphene substrate with very high native defect density estimated at $2 \times 10^{12} \mathrm{~cm}^{-2}$, which may heavily interfere with the grafting chemistry and morphology. A study by Lucchese et al. has shown a similar distortion effect of HOPG with UHV-STM around the defect sites caused by $\mathrm{Ar}^{+}$ ion beam damage. ${ }^{44}$ In addition, chemisorption of diazonium species on graphene has shown a transmission electron microscopy image revealing an expanded lattice constant due to the formation of $\mathrm{sp}^{3} \mathrm{C}-\mathrm{C}$ bonds. ${ }^{45}$ The STM results we present here are the first to show lattice distortions from $\mathrm{sp}^{3}$ defects following grafting on pristine and virtually defect-free HOPG in ambient conditions. 
The aryl species are not stable under STM scanning at the high current and low bias values required for imaging the graphite lattice $\left(I_{\text {set }}=0.1-0.3 \mathrm{nA}\right.$ and $\mathrm{V}_{\text {bias }}=-0.001$ up to $\left.-0.1 \mathrm{~V}\right)$. Consequently, the grafted structures can only be imaged once or twice while imaging the graphite lattice before being removed by the STM tip. This effect is illustrated in Figure 10: two of the clusters in Figure 10a have been labeled A and B for reference; the subsequent STM image (Figure 10b) shows that two of the original clusters have disappeared. The HOPG lattice can be imaged after removal of the clusters by the STM tip. No defects or distortions are detected, strongly suggesting that the graphite lattice reverts back to its original configuration once the aryl groups are detached. This aspect will be addressed in the next section.

Figure 10c shows a cluster imaged along with the graphite lattice for 3,5-TBD $(0.1 \mathrm{mM})$ modified HOPG. The same type of distortion at the periphery of the cluster is observed. A Fourier transform image of the distorted graphite lattice region, marked by the white dashed square, is shown in the inset. Additional spots are observed along with the hexagonal pattern of the graphite lattice, indicating a Moiré pattern distortion to the lattice. This is likely caused by a dislocation of the top layer after $\mathrm{sp}^{3}$ rehybridization of a number of sites at the cluster. The distortion to the carbon lattice caused by grafting is consistent with the observed red shift of the G- and 2D-bands in the Raman spectra of graphene due to the elongation of the carbon-carbon bonds. The next STM image taken of the same area, Figure 10d, reveals that the cluster is already removed. The Fourier transform image of the graphite lattice, shown in the inset, shows a hexagonal pattern, as expected for a pristine hexagonal carbon lattice. This is further evidence that, upon removal of the covalently attached aryl clusters by the STM tip, the carbon lattice reverts back from $\mathrm{sp}^{3}$ to $\mathrm{sp}^{2}$. 

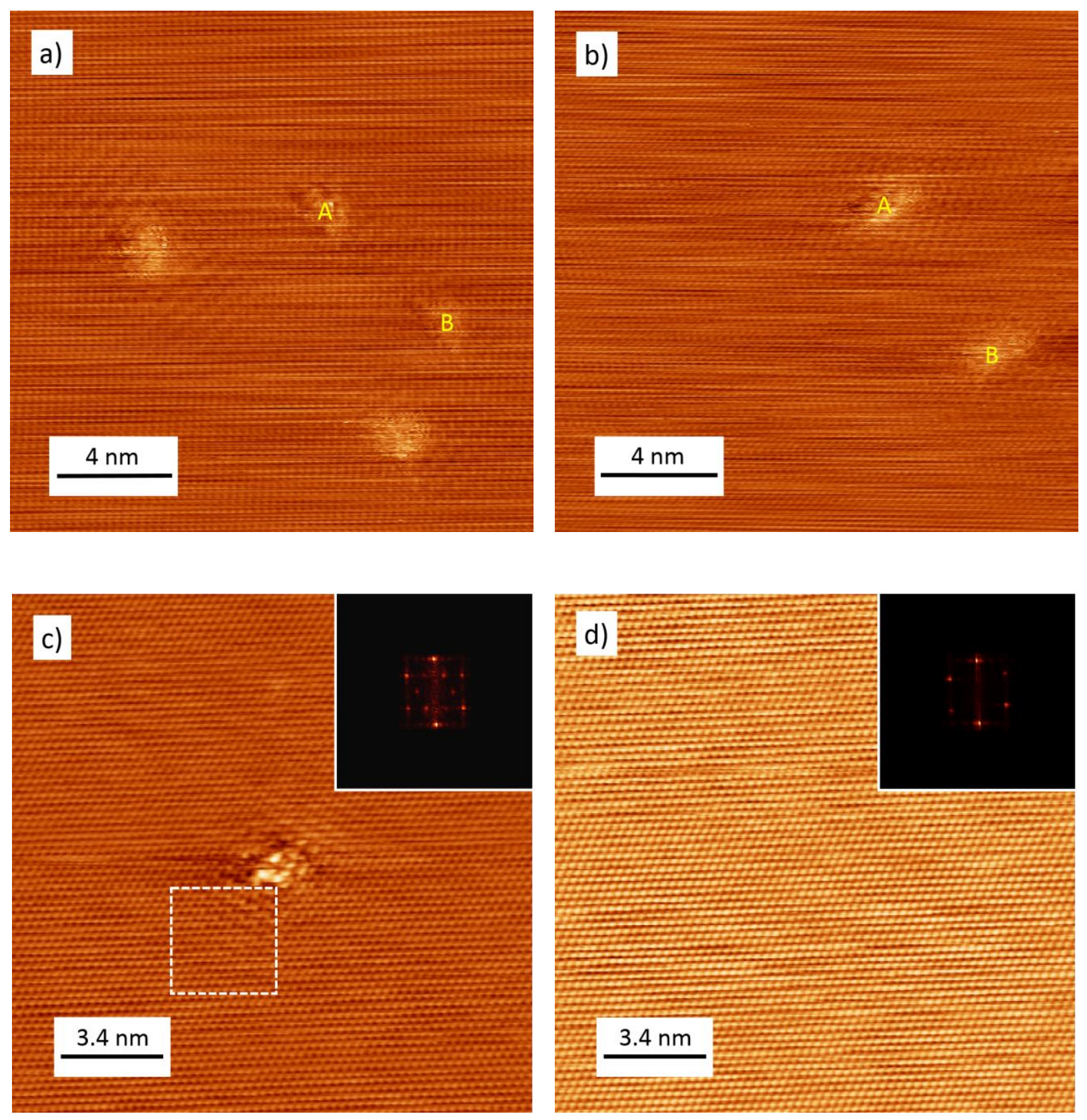

Figure 10. (a,b) Sequential high-resolution STM images of 4-NBD clusters grafted on HOPG showing a distortion of the graphite lattice. The second image shows that two clusters have been removed (A and B remain) $\left(\mathrm{I}_{\text {set }}=0.2 \mathrm{nA}, \mathrm{V}_{\text {bias }}=-0.1 \mathrm{~V}\right)$; (c,d) Sequential high-resolution STM images of 3,5-TBD grafted on HOPG. In the second image, scanning has removed the cluster and pristine HOPG is recovered $\left(\mathrm{I}_{\mathrm{set}}=0.1 \mathrm{nA}, \mathrm{V}_{\text {bias }}=-0.01 \mathrm{~V}\right)$. Fourier transforms are shown as inset. 
The removal of the spots by STM scanning, and therefore of the grafted species, opens the way for controlled nano-manipulation using STM. As described previously, the grafted species we observe are not stable to scanning, and are gradually removed at a current value of $0.08 \mathrm{nA}$ or higher. The STM image in Figure 11a shows the 4-NBD modified HOPG surface immediately after the central area $(200 \times 200 \mathrm{~nm})$ was scanned at $0.5 \mathrm{nA}$ consecutively several times. The grafted species were also removed from graphene on $\mathrm{Cu}$ after sequential scanning, as shown in Figure 11b. Instantaneous removal of the clusters could be achieved by scanning at high current and low bias ( $1 \mathrm{mV}, 0.3 \mathrm{nA})$. High current and low bias scanning also immediately removes the 3,5-TBD clusters. Due to the high density of grafted species in this case, complex shapes and patterns can be created by nanoshaving of clusters in designated areas. An example is shown in Figure 11c, where 'Nano-man' was drawn by scanning of the designated areas using the stated tunneling parameters. Figure 11d finally shows that it is also possible to selectively remove the 3,5-TBD clusters from graphene.
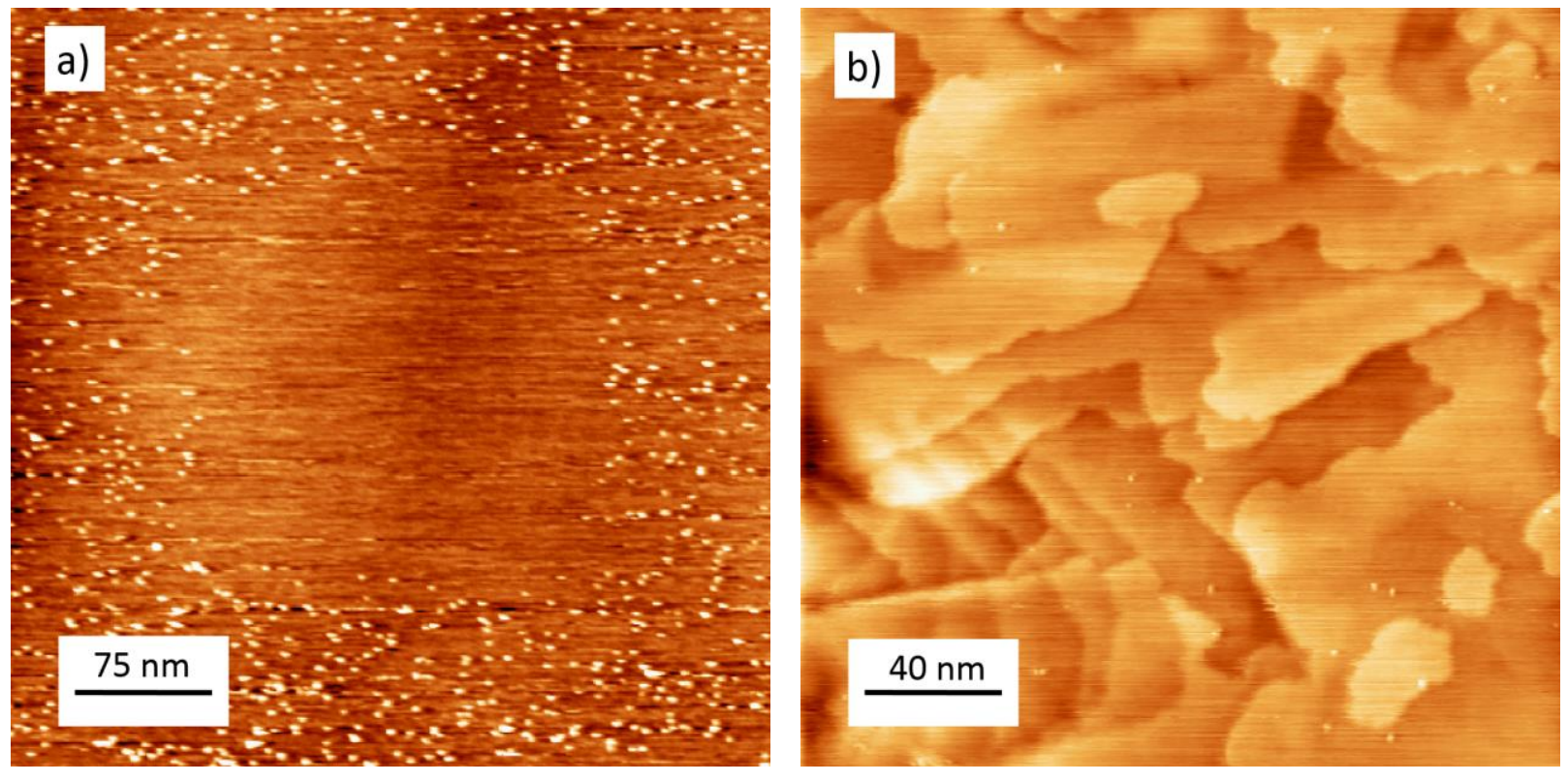

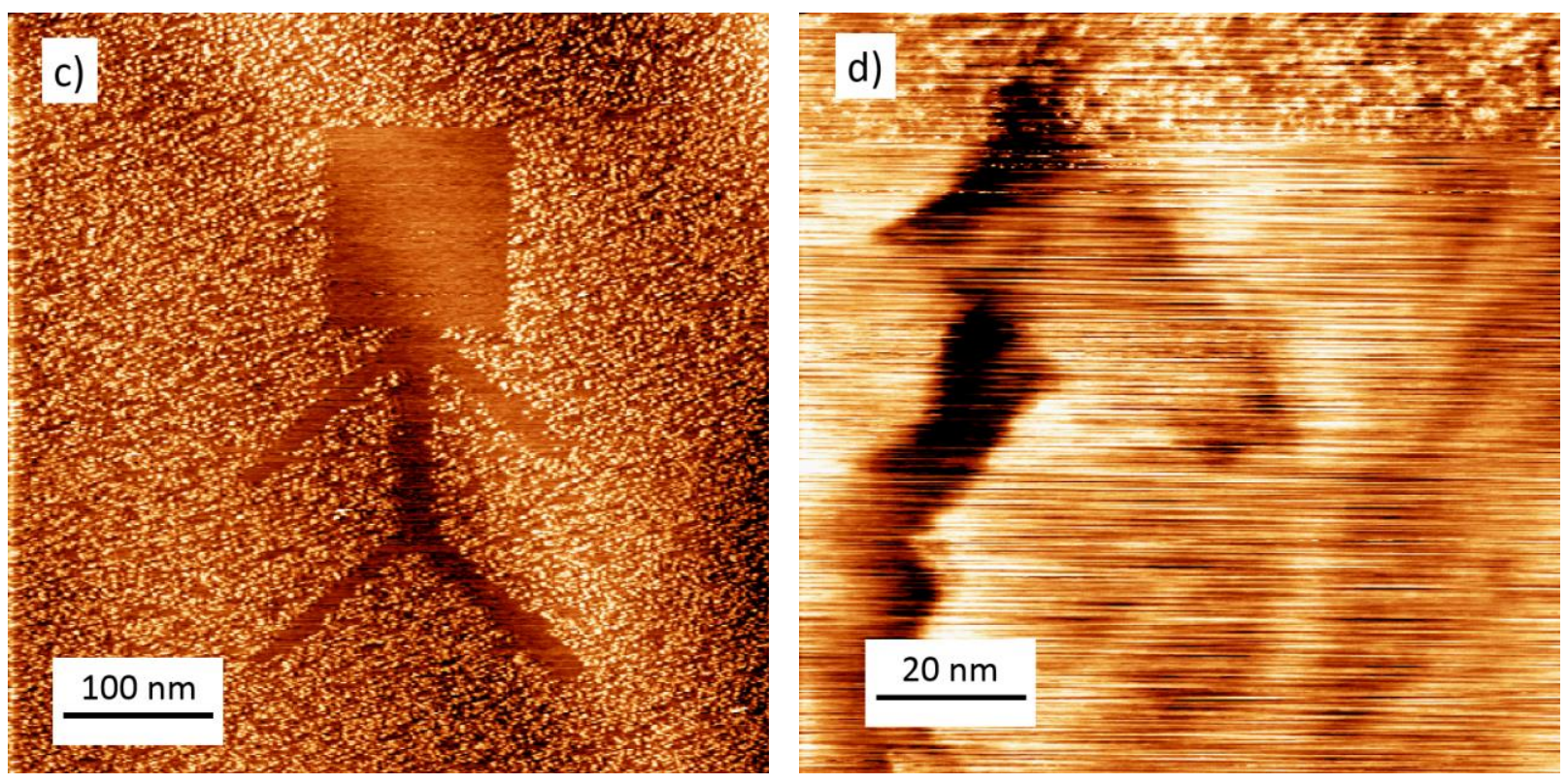

Figure 11. (a) STM image immediately after nanoshaving of grafted 4-NBD clusters from the central 200 x $200 \mathrm{~nm}^{2}$ area on HOPG; (b) STM image of 4-NBD grafted on CVD graphene on Cu after nanoshaving; (c) Nanoshaving of 3,5-TBD grafted on HOPG, creating 'Nano-man' (d) Nanoshaving of 3,5-TBD grafted on CVD graphene on $\mathrm{Cu}$. Tunneling parameters (a) $\mathrm{I}_{\text {set }}=0.08$ $\mathrm{nA}, \mathrm{V}_{\text {bias }}=-0.4 \mathrm{~V} ;(\mathrm{b}, \mathrm{c}, \mathrm{d}) \mathrm{I}_{\text {set }}=0.1 \mathrm{nA}, \mathrm{V}_{\text {bias }}=-0.4 \mathrm{~V}$

In order to prove conclusively that STM nanoshaving of grafted aryl groups restores the pristine $\mathrm{sp}^{2}$ carbon lattice, we combined nanoshaving with confocal Raman spectroscopy to detect the nanopatterns spectroscopically. Due to the relatively larger D-band intensity compared to 4-NBD, we limit ourselves to 3,5-TBD on HOPG in this section. 3,5-TBD was grafted electrochemically onto HOPG. The following experiments involved positioning the STM tip, in this case made of tungsten, over a designated scanning area. The area was marked by a laser spot, approximately 1 $\mu \mathrm{m}$ in diameter. Laser light illumination did not have any effect on the D-band intensity decay. Raman spectra were measured continuously for 1.5 hours, but the D-band intensity did not show 
any significant change. The focused laser spot at the HOPG surface was set at $1 \mu \mathrm{m}$ distance from the W-tip apex, as is illustrated by the confocal Raman mapping image of the STM tip above the scanning area, Figure 12a.

Upon approach to the surface, the STM tip scanned three sets of areas, $1 \times 20 \mu \mathrm{m}$, for 7 times at each position, as illustrated in Figure $12 \mathrm{~b}(1 \mathrm{nA}, 0.1 \mathrm{~V})$. The sample was subsequently scanned by confocal Raman D-band mapping. Figure 12c shows the confocal Raman map plotted by D/Gband intensity ratio. The integration time of each pixel was $1.5 \mathrm{~s}$. The image clearly shows a D/Gband intensity ratio decrease at the three corresponding channels in the areas where the STM tip had repeatedly scanned. In addition, Figure 12d shows two sets of Raman spectra superimposed onto one another, taken along one of the designated scanning areas before and after STM scanning. Before STM scanning, there is a relatively large D-band observed at $1330 \mathrm{~cm}^{-1}$, as well as the Gband at $1576 \mathrm{~cm}^{-1}$. After repeated STM scanning, the D-band is no longer observed, analogous to the confocal Raman mapping results shown in Figure 12c. In addition, G-band intensity was slightly higher than D-band, clearly indicating that $\mathrm{sp}^{3}$ rehybridizes to $\mathrm{sp}^{2}$ by scanning. A stronger G-band should be observed in a G-band confocal mapping image, but a clear dependence could not be observed due to too limited a change. These results agree with the conclusion that the aryl radical species from 3,5-TBD are covalently grafted to the HOPG basal plane. The confocal Raman image provides spectroscopic evidence, in addition to the STM topographic evidence, of patterned removal of grafted species.

We also attempted the same procedure on 3,5-TBD (1 mM) functionalized graphene. However, the experiments have been so far unsuccessful, resulting in a large D-band at the scratched areas as a result of tip damage to the graphene layer. Due to the polycrystalline nature of the $\mathrm{Cu}$ substrate, the surface is quite rough (see supplementary information, Figure S4b, for a large scale STM image 
of bare graphene). Consequently, nanoshaving by scanning at high current and low bias over the micrometer scale required for confocal Raman mapping is not trivial. This should be possible on graphene on a more planar substrate, such as $\mathrm{SiC}$ or h-BN.
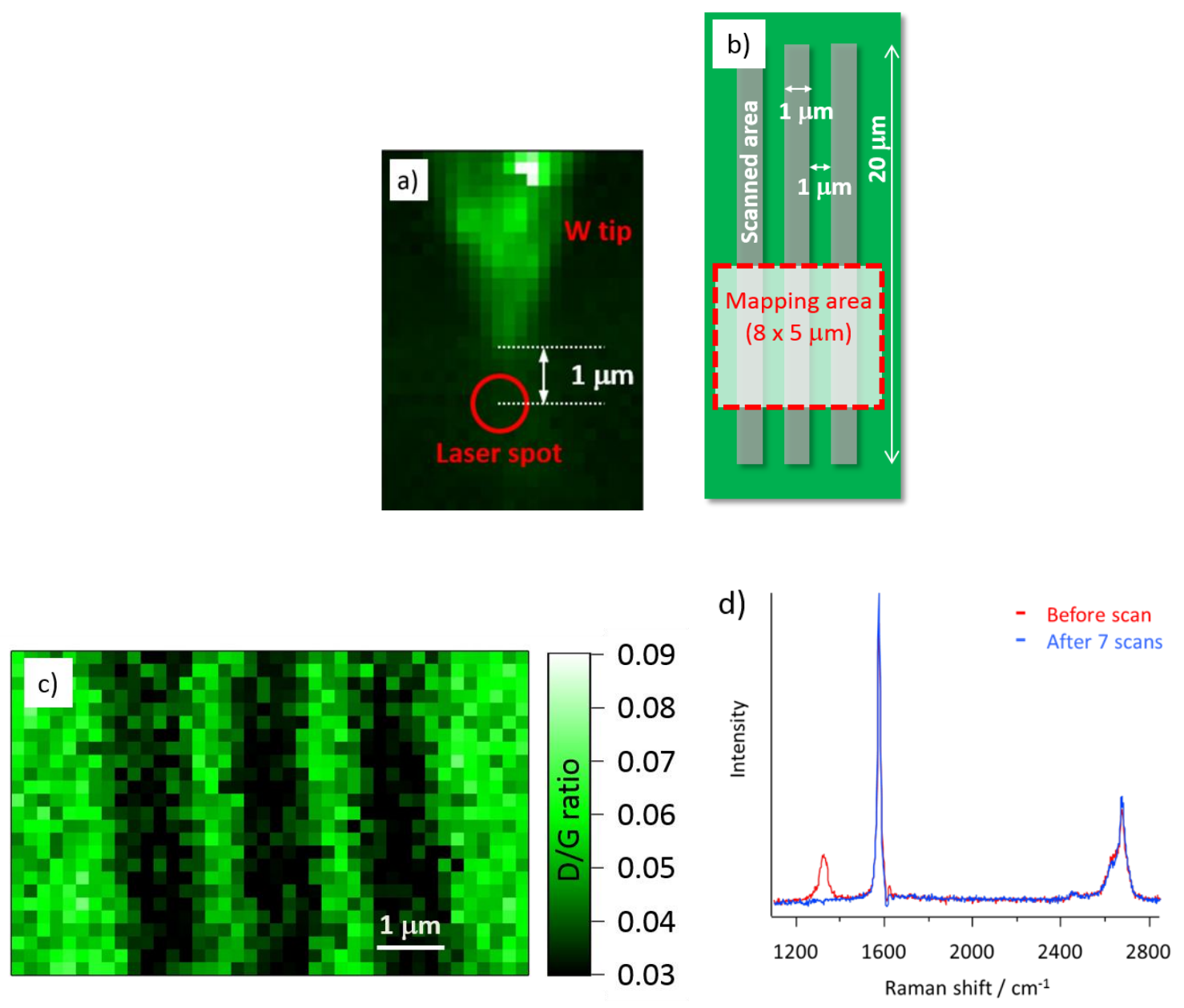

Figure 12. (a) Confocal Raman map of the STM tip above the scanning area; (b) Scheme showing STM scanned (7 times at each position) and Raman mapped areas; (c) confocal Raman D-band map of STM scanned areas, (d) Raman spectra before (red) and after (blue) STM nanoshaving.

A final application has been discovered for the nanopatterned structures formed in the 3,5-TBD grafted film. When $n$-pentacontane $\left(n-\mathrm{C}_{50} \mathrm{H}_{102}\right)$ is deposited in phenyloctane solution on the grafted 
HOPG sample, in situ nanoshaving at the liquid-solid interface results in the exposed surface becoming host to $n$-pentacontane self-assembly. Self-assembly selectively occurs in the exposed regions and not on top of, or in between, the grafted aryl species. Figure 13a shows an STM image of a series of nanotrenches within the grafted film, created by the STM tip. $n$-Pentacontane is seen to form multiple domains of parallel lamellae in the exposed area. Figure 13b shows a close-up view of a high-resolution image of a series of $n$-pentacontane rows. The image for a single pentacontane domain was taken from a separate location to that shown in Figure 13a. The $n$ $\mathrm{C}_{50} \mathrm{H}_{102}$ molecules self-assemble as they do on bare $\mathrm{HOPG},{ }^{46}$ indicating there is no damage to the surface carbon lattice upon removal of the grafted molecules. An illustration of STM tip nanoshaving and molecular self-assembly within the exposed area is presented in Figure 13c. Despite the fact that the grafted clusters can be removed by the STM tip during scanning they do not degrade over time. Modified HOPG samples as much as a month old were scanned with STM and showed no alteration with respect to newer samples. In addition, the robustness of modified samples to solvent is self-evident in the STM images of $n$-pentacontane in phenyloctane, showing no disruption to the grafted clusters in areas without tip manipulation. 1,2,4-Trichlorobenzene (TCB) was also used on the grafted samples, again showing no degradation. Therefore, since modified samples are stable over time and can withstand further processing involving solvents, we envisage covalent modification to hold great potential for applications. 

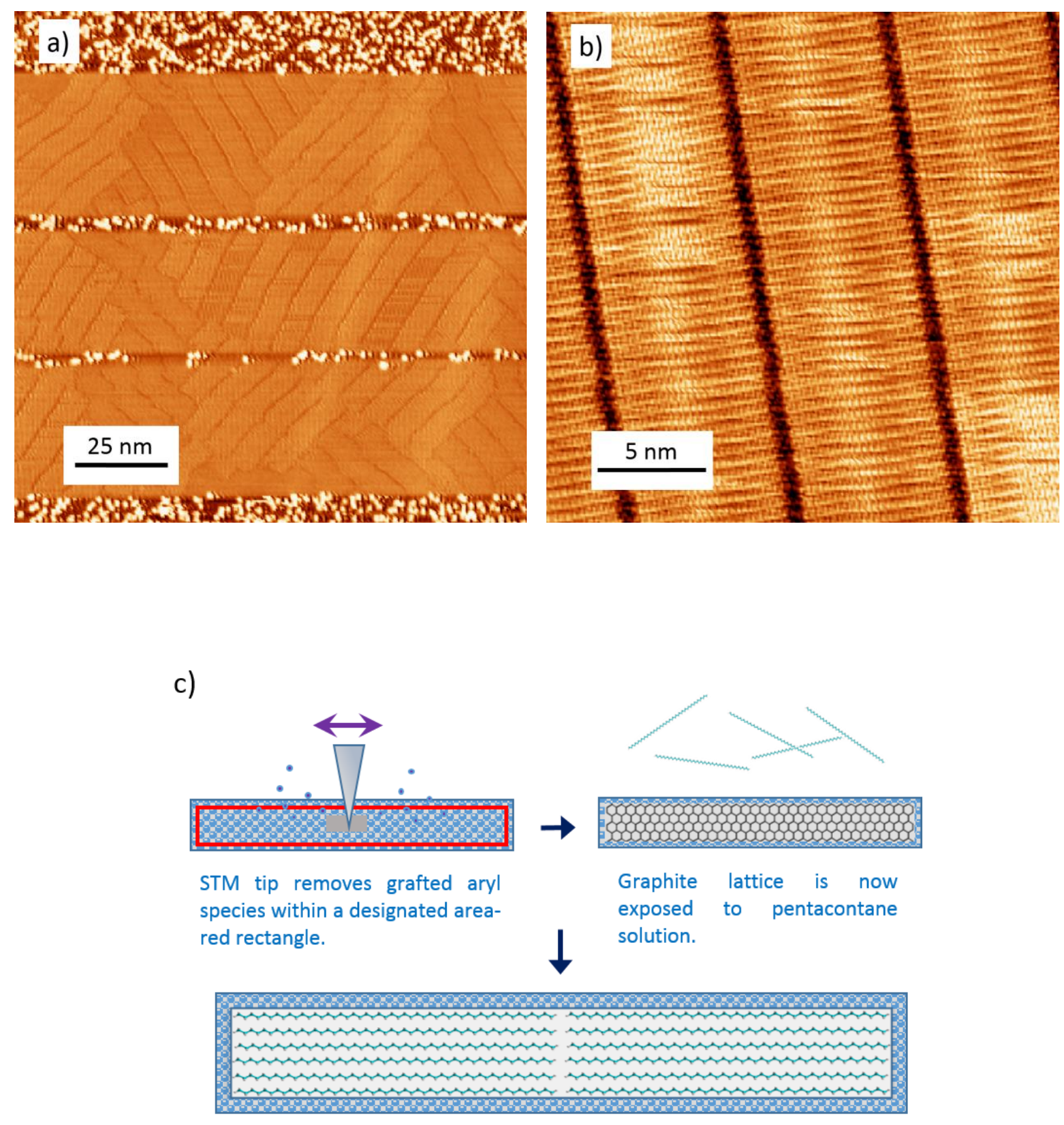

Pentacontane self-assembly can selectively occur at the exposed graphite lattice.

Figure 13. (a) STM image of $n$-pentacontane $\left(n-\mathrm{C}_{50} \mathrm{H}_{102}\right)$ self-assembly within nanotrenches of bare HOPG produced by nanoshaving of grafted 3,5-TBD, forming lamellar structures; (b) Highresolution STM image of $n$-pentacontane; (c) Scheme of STM nanoshaving, followed by $n$ - 
pentacontane self-assembly within the exposed area. Tunneling parameters (a) $I_{\text {set }}=0.165 \mathrm{nA}$, $\mathrm{V}_{\text {bias }}=+0.6 \mathrm{~V}$; (b) $\mathrm{I}_{\text {set }}=0.18 \mathrm{nA}, \mathrm{V}_{\text {bias }}=+0.45 \mathrm{~V}$.

\section{Conclusions}

We have presented conclusive evidence that on electrochemical grafting of diazonium compounds to graphene and HOPG, covalent attachment with $\mathrm{sp}^{3}$ rehybridization of the surface site takes place. By selecting sterically hindered reagents, grafting can be limited to a monolayer, achieving a high density. Raman spectroscopy also indicates tunable band-gap opening of graphene following grafting of 3,5-TBD by varying the diazonium reagent concentration.

We have used high resolution STM measurements to directly observe graphite lattice buckling at the site of covalent attachment to a previously defect-free HOPG surface for the first time, demonstrating the value of STM for characterizing similar systems.

Using suitable tunneling parameters, we have demonstrated STM nanoshaving of grafted molecules for the first time under ambient conditions, which allows complex topographic patterns to be created in high density grafted layers. Importantly, using Raman spectroscopy and STM, we

have proven that the pristine $\mathrm{sp}^{2}$ carbon lattice is restored on removal of grafted species. After nanoshaving, molecular self-assembly of $n$-pentacontane takes place in the same manner as on HOPG that was never modified, further testifying pristine graphite recovery.

Our results settle several long-standing controversies regarding the details of diazonium modification of carbon surfaces, while high density monolayer grafting opens the way for efficient graphene-based devices. The nano-manipulation protocol will be very useful for nanostructuring surfaces to study, for instance, self-assembly under nanoconfinement conditions. 


\section{Methods}

All STM experiments were performed using a Molecular Imaging STM system, operating in constant-current mode. STM tips were prepared by mechanical cutting of Pt/Ir wire $(80 \% / 20 \%$, diameter $0.25 \mathrm{~mm}$ ). The bias voltage refers to the substrate. For AFM measurements, a Multimode AFM with a Nanoscope IV controller (Veeco/Digital Instruments) was employed in intermittent contact mode. Substrates consisted of HOPG (grade ZYB, Advanced Ceramics Inc., Cleveland, $\mathrm{OH})$. Graphene samples were obtained from Graphenea, and consisted of CVD grown graphene on $\mathrm{Cu}$ foil. STM and AFM data analysis was performed using WSxM 5.0. ${ }^{40}$

4-Nitrobenzenediazonium (4-NBD) tetrafluoroborate (97\%) and analytical grade hydrochloric and perchloric acid were purchased from Sigma-Aldrich and used without further purification. High purity water (Milli-Q, Millipore, $18.2 \mathrm{M} \Omega \mathrm{cm}$, TOC $<3 \mathrm{ppb}$ ) was used throughout. Electrolyte solutions were deoxygenated with argon gas (grade 5.0, Praxair) for several hours before use. 3,5Bis-tert-butylbenzenediazonium (3,5-TBD) is unstable, and decomposes rapidly; hence, it was synthesized from the aniline precursor immediately prior to electrochemical reduction. 3,5-Bistert-butylaniline (98\%) was purchased from TCI-Tokyo Chemical Industry Co., Ltd. and used without further purification. 3,5-TBD solutions with concentrations between 0.1 and $10 \mathrm{mM}$ were prepared by dissolving the desired amount of 3,5-bis-tert-butylaniline in $\mathrm{HCl}_{\mathrm{aq}}(0.5 \mathrm{M})$; aqueous $\mathrm{NaNO}_{2}(0.1 \mathrm{~mL}, 1 \mathrm{M})$ was mixed with $5 \mathrm{~mL}$ amine solution approximately 3 minutes prior to injection into the electrochemical cell.

All electrochemical measurements were performed using an Autolab PGSTAT101 potentiostat (Metrohm-Autolab BV, The Netherlands). Prior to each experiment, the HOPG electrode was freshly cleaved using scotch tape. The electrochemical modification of the HOPG and graphene samples was carried out in a lab-built single-compartment three-electrode cell, with a working 
electrode area of $38.5 \mathrm{~mm}^{2}$, $\mathrm{Pt}$ wire counter and $\mathrm{Ag} / \mathrm{AgCl} / 3 \mathrm{M} \mathrm{NaCl}$ reference electrodes. During the measurements, the electrolyte was kept under a solvent-saturated Ar (grade 5.0, Praxair) atmosphere. After modification, the 4-NBD and 3,5-TBD modified HOPG and graphene samples were rinsed with Milli-Q-water to remove any physisorbed material from the surface, and dried in a stream of argon.

Raman measurements were performed with an OmegaScopeTM 1000 (AIST-NT). Laser light $(632.8 \mathrm{~nm})$ from a He-Ne laser was focused onto the sample surface from the side (with an angle of $28^{\circ}$ to sample surface) and top, for 'grating' and 'normal' measurements, respectively, through an objective (MITUTOYO, BD Plan Apo 100x, N.A. 0.7). Optical density at sample surface was

about $500 \mathrm{~kW} / \mathrm{cm}^{2}$. Raman scattering was collected with the same objective and directed to a Raman spectrograph (Horiba JY, iHR-320) equipped with a cooled-charge coupled device (CCD) camera operated at $-100{ }^{\circ} \mathrm{C}$ (Andor technology, DU920P-BRDD) through a pinhole, a dichroic mirror (Chroma Technology Corporation, Z633RDC) and long pass filter (Chroma Technology Corporation, HQ645LP). Accumulation time for each point in 'grating' measurement was $1 \mathrm{~s}$. All of the measurements were carried out under ambient conditions and at room temperature.

\section{Acknowledgements}

This work is supported by the Fund of Scientific Research- Flanders (FWO), KU Leuven (GOA 11/003), Belgian Federal Science Policy Office (IAP-7/05), and DFG Priority Program SPP 1355, SENSOR. The research leading to these results has also received funding from the European Research Council under the European Union's Seventh Framework Programme (FP7/20072013)/ERC Grant Agreement no. 340324 to SDF and PLASMHACAT (no. 280064) to HU, and the Japan Science and Technology Agency PRESTO program to H.U. W.V. thanks FWO for a postdoctoral fellowship. W. F. thanks IWT for a PhD fellowship. 
Supporting Information Available: Extra CV, STM, AFM, and Raman spectroscopy results. This material is available free of charge via the Internet at http://pubs.acs.org.

\section{References}

1. Novoselov, K. S.; Geim, A. K.; Morozov, S. V.; Jiang, D.; Zhang, Y.; Dubonos, S. V.; Grigorieva, I. V.; Firsov, A. A., Electric Field Effect in Atomically Thin Carbon Films. Science 2004, 306, 666-669.

2. Avouris, P., Graphene: Electronic and Photonic Properties and Devices. Nano Lett. 2010, $10,4285-4294$.

3. Niyogi, S.; Bekyarova, E.; Itkis, M. E.; Zhang, H.; Shepperd, K.; Hicks, J.; Sprinkle, M.; Berger, C.; Lau, C. N.; Deheer, W. A., et al., Spectroscopy of Covalently Functionalized Graphene. Nano Lett. 2010, 10, 4061-4066.

4. Hossain, M. Z.; Walsh, M. A.; Hersam, M. C., Scanning Tunneling Microscopy, Spectroscopy, and Nanolithography of Epitaxial Graphene Chemically Modified with Aryl Moieties. J. Am. Chem. Soc. 2010, 132, 15399-15403.

5. Bekyarova, E.; Itkis, M. E.; Ramesh, P.; Berger, C.; Sprinkle, M.; de Heer, W. A.; Haddon, R. C., Chemical Modification of Epitaxial Graphene: Spontaneous Grafting of Aryl Groups. J. Am. Chem. Soc. 2009, 131, 1336-1337.

6. Schedin, F.; Geim, A. K.; Morozov, S. V.; Hill, E. W.; Blake, P.; Katsnelson, M. I.; Novoselov, K. S., Detection of Individual Gas Molecules Adsorbed on Graphene. Nat. Mater. 2007, 6, 652-655.

7. Kulkarni, G. S.; Reddy, K.; Zhong, Z.; Fan, X., Graphene Nanoelectronic Heterodyne Sensor for Rapid and Sensitive Vapour Detection. Nat. Commun. 2014, 5. 
8. Englert, J. M.; Dotzer, C.; Yang, G.; Schmid, M.; Papp, C.; Gottfried, J. M.; Steinrück, H.-P.; Spiecker, E.; Hauke, F.; Hirsch, A., Covalent Bulk Functionalization of Graphene. Nat. Chem. 2011, 3, 279-286.

9. Singh, A. K.; Yakobson, B. I., Electronics and Magnetism of Patterned Graphene Nanoroads. Nano Lett. 2009, 9, 1540-1543.

10. Sessi, P.; Guest, J. R.; Bode, M.; Guisinger, N. P., Patterning Graphene at the Nanometer Scale Via Hydrogen Desorption. Nano Lett. 2009, 9, 4343-4347.

11. de Andres, P. L.; Verges, J. A., First-Principles Calculation of the Effect of Stress on the Chemical Activity of Graphene. Appl. Phys. Lett. 2008, 93, 3.

12. Elias, D. C.; Nair, R. R.; Mohiuddin, T. M. G.; Morozov, S. V.; Blake, P.; Halsall, M. P.; Ferrari, A. C.; Boukhvalov, D. W.; Katsnelson, M. I.; Geim, A. K., et al., Control of Graphene's Properties by Reversible Hydrogenation: Evidence for Graphane. Science 2009, 323, 610-613.

13. Combellas, C.; Kanoufi, F.; Pinson, J.; Podvorica, F. I., Sterically Hindered Diazonium Salts for the Grafting of a Monolayer on Metals. J. Am. Chem. Soc. 2008, 130, 85768577.

14. Combellas, C.; Jiang, D. E.; Kanoufi, F.; Pinson, J.; Podvorica, F. I., Steric Effects in the Reaction of Aryl Radicals on Surfaces. Langmuir 2009, 25, 286-293.

15. Ma, H. F.; Lee, L.; Brooksby, P. A.; Brown, S. A.; Fraser, S. J.; Gordon, K. C.; Leroux, Y. R.; Hapiot, P.; Downard, A. J., Scanning Tunneling and Atomic Force Microscopy Evidence for Covalent and Noncovalent Interactions between Aryl Films and Highly Ordered Pyrolytic Graphite. J. Phys. Chem. C 2014, 118, 5820-5826. 
16. Adenier, A.; Combellas, C.; Kanoufi, F.; Pinson, J.; Podvorica, F. I., Formation of Polyphenylene Films on Metal Electrodes by Electrochemical Reduction of Benzenediazonium Salts. Chem. Mater. 2006, 18, 2021-2029.

17. Leroux, Y. R.; Fei, H.; Noel, J. M.; Roux, C.; Hapiot, P., Efficient Covalent Modification of a Carbon Surface: Use of a Silyl Protecting Group to Form an Active Monolayer. $J$. Am. Chem. Soc. 2010, 132, 14039-14041.

18. Malmos, K.; Dong, M. D.; Pillai, S.; Kingshott, P.; Besenbacher, F.; Pedersen, S. U.; Daasbjerg, K., Using a Hydrazone-Protected Benzenediazonium Salt to Introduce a nearMonolayer of Benzaldehyde on Glassy Carbon Surfaces. J. Am. Chem. Soc. 2009, 131, 4928-4936.

19. Menanteau, T.; Levillain, E.; Breton, T., Spontaneous Grafting of Nitrophenyl Groups on Carbon: Effect of Radical Scavenger on Organic Layer Formation. Langmuir 2014, 30 , 7913-7918.

20. Shen, C.; Buck, M., Nanoscale Patterning of a Self-Assembled Monolayer by Modification of the Molecule-Substrate Bond. Beilstein J. Nanotechnol. 2014, 5, 258267.

21. Sinitskii, A.; Dimiev, A.; Corley, D. A.; Fursina, A. A.; Kosynkin, D. V.; Tour, J. M., Kinetics of Diazonium Functionalization of Chemically Converted Graphene Nanoribbons. ACS Nano 2010, 4, 1949-1954.

22. Koehler, F. M.; Luechinger, N. A.; Ziegler, D.; Athanassiou, E. K.; Grass, R. N.; Rossi, A.; Hierold, C.; Stemmer, A.; Stark, W. J., Permanent Pattern-Resolved Adjustment of the Surface Potential of Graphene-Like Carbon through Chemical Functionalization. Angew. Chem., Int. Ed. 2009, 48, 224-227. 
23. Kirkman, P. M.; Guell, A. G.; Cuharuc, A. S.; Unwin, P. R., Spatial and Temporal Control of the Diazonium Modification of $\mathrm{Sp}^{2}$ Carbon Surfaces. J. Am. Chem. Soc. 2014, $136,36-39$.

24. Wang, Q. H.; Jin, Z.; Kim, K. K.; Hilmer, A. J.; Paulus, G. L. C.; Shih, C.-J.; Ham, M.H.; Sanchez-Yamagishi, J. D.; Watanabe, K.; Taniguchi, T., et al., Understanding and Controlling the Substrate Effect on Graphene Electron-Transfer Chemistry Via Reactivity Imprint Lithography. Nat. Chem. 2012, 4, 724-732.

25. Baranton, S.; Belanger, D., Electrochemical Derivatization of Carbon Surface by Reduction of in Situ Generated Diazonium Cations. J. Phys. Chem. B 2005, 109, 2440124410.

26. Rappich, J.; Merson, A.; Roodenko, K.; Dittrich, T.; Gensch, M.; Hinrichs, K.; Shapira, Y., Electronic Properties of Si Surfaces and Side Reactions During Electrochemical Grafting of Phenyl Layers. J. Phys. Chem. B 2006, 110, 1332-1337.

27. Haque, A. J.; Kim, K., Reusable Bio-Functionalized Surfaces Based on Electrochemical Desorption of Benzenediazonium-Grafted Organic Layers. Chem. Commun. 2011, 47, $6855-6857$.

28. Ferrari, A. C.; Robertson, J., Interpretation of Raman Spectra of Disordered and Amorphous Carbon. Phys. Rev. B 2000, 61, 14095-14107.

29. Malard, L. M.; Pimenta, M. A.; Dresselhaus, G.; Dresselhaus, M. S., Raman Spectroscopy in Graphene. Phys. Rep. 2009, 473, 51-87.

30. Ni, Z. H.; Yu, T.; Lu, Y. H.; Wang, Y. Y.; Feng, Y. P.; Shen, Z. X., Uniaxial Strain on Graphene: Raman Spectroscopy Study and Band-Gap Opening. ACS Nano 2008, 2, 23012305. 
31. Yu, T.; Ni, Z.; Du, C.; You, Y.; Wang, Y.; Shen, Z., Raman Mapping Investigation of Graphene on Transparent Flexible Substrate: The Strain Effect. J. Phys. Chem. C 2008, $112,12602-12605$.

32. Zhou, S. Y.; Gweon, G. H.; Fedorov, A. V.; First, P. N.; de Heer, W. A.; Lee, D. H.; Guinea, F.; Castro Neto, A. H.; Lanzara, A., Substrate-Induced Bandgap Opening in Epitaxial Graphene. Nat. Mater. 2007, 6, 770-775.

33. Ribeiro, R. M.; Peres, N. M. R.; Coutinho, J.; Briddon, P. R., Inducing Energy Gaps in Monolayer and Bilayer Graphene: Local Density Approximation Calculations. Phys. Rev. $B$ 2008, 78, 075442.

34. Giovannetti, G.; Khomyakov, P. A.; Brocks, G.; Kelly, P. J.; van den Brink, J., SubstrateInduced Band Gap in Graphene on Hexagonal Boron Nitride: Ab Initio Density Functional Calculations. Phys. Rev. B 2007, 76, 073103.

35. Johns, J. E.; Hersam, M. C., Atomic Covalent Functionalization of Graphene. Acc. Chem. Res. 2013, 46, 77-86.

36. Bruna, M.; Ott, A. K.; Ijäs, M.; Yoon, D.; Sassi, U.; Ferrari, A. C., Doping Dependence of the Raman Spectrum of Defected Graphene. ACS Nano 2014, 8, 7432-7441.

37. Nourbakhsh, A.; Cantoro, M.; Li, B.; Müller, R.; De Feyter, S.; Heyns, M. M.; Sels, B. F.; De Gendt, S., Tunable N- and P-Type Doping of Single-Layer Graphene by Engineering Its Interaction with the Sio2 Support. Phys. Status Solidi RRL 2012, 6, 53 55.

38. Pembroke, E.; Ruan, G.; Sinitskii, A.; Corley, D.; Yan, Z.; Sun, Z.; Tour, J., Effect of Anchor and Functional Groups in Functionalized Graphene Devices. Nano Res. 2013, 6, $138-148$. 
39. Solis-Fernandez, P.; Bissett, M. A.; Tsuji, M.; Ago, H., Tunable Doping of Graphene Nanoribbon Arrays by Chemical Functionalization. Nanoscale 2015, 7, 3572-3580.

40. Horcas, I.; Fernandez, R.; Gomez-Rodriguez, J. M.; Colchero, J.; Gomez-Herrero, J.; Baro, A. M., Wsxm: A Software for Scanning Probe Microscopy and a Tool for Nanotechnology. Rev. Sci. Instrum. 2007, 78, 8.

41. Brooksby, P. A.; Downard, A. J., Electrochemical and Atomic Force Microscopy Study of Carbon Surface Modification Via Diazonium Reduction in Aqueous and Acetonitrile Solutions. Langmuir 2004, 20, 5038-5045.

42. Mertens, S. F. L.; Bütikofer, A.; Siffert, L.; Wandlowski, T., Covalent Versus Electrostatic Strategies for Nanoparticle Immobilisation. Electroanalysis 2010, 22, 29402946.

43. Stevenson, K. J.; Veneman, P. A.; Gearba, R. I.; Mueller, K. M.; Holliday, B. J.; Ohta, T.; Chan, C. K., Controlled Covalent Modification of Epitaxial Single Layer Graphene on 6h-Sic (0001) with Aryliodonium Salts Using Electrochemical Methods. Faraday Discuss. 2014, 172, 273-291.

44. Lucchese, M. M.; Stavale, F.; Ferreira, E. H. M.; Vilani, C.; Moutinho, M. V. O.; Capaz, R. B.; Achete, C. A.; Jorio, A., Quantifying Ion-Induced Defects and Raman Relaxation Length in Graphene. Carbon 2010, 48, 1592-1597.

45. Seo, S.; Min, M.; Lee, S. M.; Lee, H., Photo-Switchable Molecular Monolayer Anchored between Highly Transparent and Flexible Graphene Electrodes. Nat. Commun. 2013, 4 , 1920. 
46. Piot, L.; Marchenko, A.; Wu, J. S.; Mullen, K.; Fichou, D., Structural Evolution of HexaPeri-Hexabenzocoronene - Adlayers in Heteroepitaxy on N-Pentacontane Template Monolayers. J. Am. Chem. Soc. 2005, 127, 16245-16250.

\section{Table of Contents}
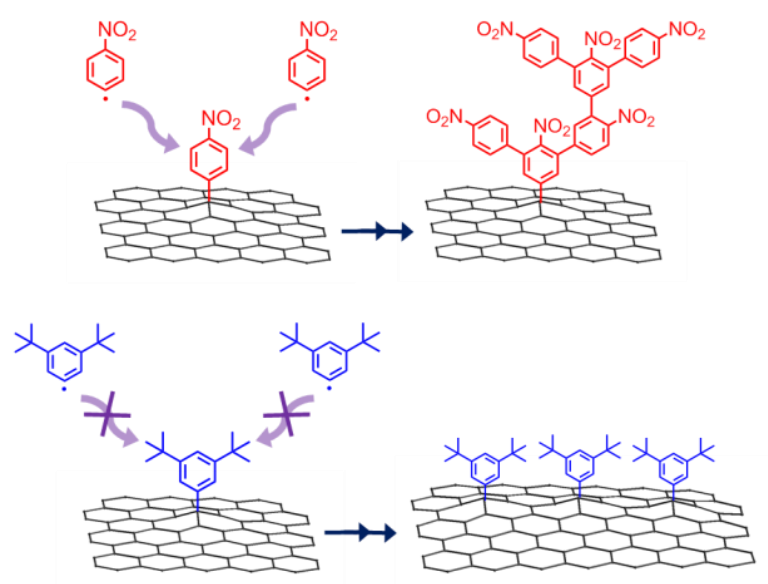